\title{
Sticky orbits in a kicked-oscillator model
}

\author{
J. H. Lowenstein†, G. Poggiaspalla, and F. Vivaldi \\ $\dagger$ Dept. of Physics, New York University, 2 Washington Place, New York, NY 10003, USA \\ School of Mathematical Sciences, Queen Mary, University of London, London E1 4NS, UK
}

\begin{abstract}
We study a 4-fold symmetric kicked-oscillator map with sawtooth kick function. For the values of the kick amplitude $\lambda=2 \cos (2 \pi p / q)$ with rational $p / q$, the dynamics is known to be pseudochaotic, with no stochastic web of non-zero Lebesgue measure. We show that this system can be represented as a piecewise affine map of the unit square - the so-called local map - driving a lattice map. We develop a framework for the study of long-time behaviour of the orbits, in the case in which the local map features exact scaling. We apply this method to several quadratic irrational values of $\lambda$, for which the local map possesses a full Legesgue measure of periodic orbits; these are promoted to either periodic orbits or accelerator modes of the kickedoscillator map. By constrast, the aperiodic orbits of the local map can generate various asymptotic behaviours. For some parameter values the orbits remain bounded, while others have excursions which grow logarithmically or as a power of the time. In the power-law case, we derive rigorous criteria for asymptotic scaling, governed by the largest eigenvalue of a recursion matrix. We illustrate the various behaviours by performing exact calculations with algebraic numbers; the hierarchical nature of the symbolic dynamics allows us to sample extremely long orbits with high efficiency, i.e., uniformly on a logarithmic time scale.
\end{abstract}

April 27, 2005 


\section{Introduction}

The boundary between regular and chaotic motions in hamiltonian systems has been studied for two decades [7,30]. In a generic system, which is neither integrable nor ergodic, chaotic orbits are strongly unstable near the centre of the chaotic regions, and near-stable in the vicinity of the boundary, with these two regimes alternating irregularly. It has been known for a long time that the intricate structure of the islands of stability near the boundary affects transport and correlation decay [7,23]. This phenomenon is particularly prominent in a class of systems characterized by extended networks of thin chaotic layers, where numerical simulations reveal orbits which have lengthy sojourns near islands of stability — so-called sticky orbits - interspersed with rapid flights within a chaotic layer, leading to strongly anomalous diffusion and fractional kinetics [37-39].

It is possible to construct deterministic models which feature only boundary-type dynamics; these models are necessarily non-generic (invariably they are discontinuous), and include billiards as well as discontinuous kicked-oscillator models $[29,40,41]$. Their Lyapunov exponents are zero, but, through a mechanism of branching without stretching generated by the discontinuity, they do reproduce the skeleton of the complex phase-space structures which one associates with the chaos border (see figure 1). This phenomenon

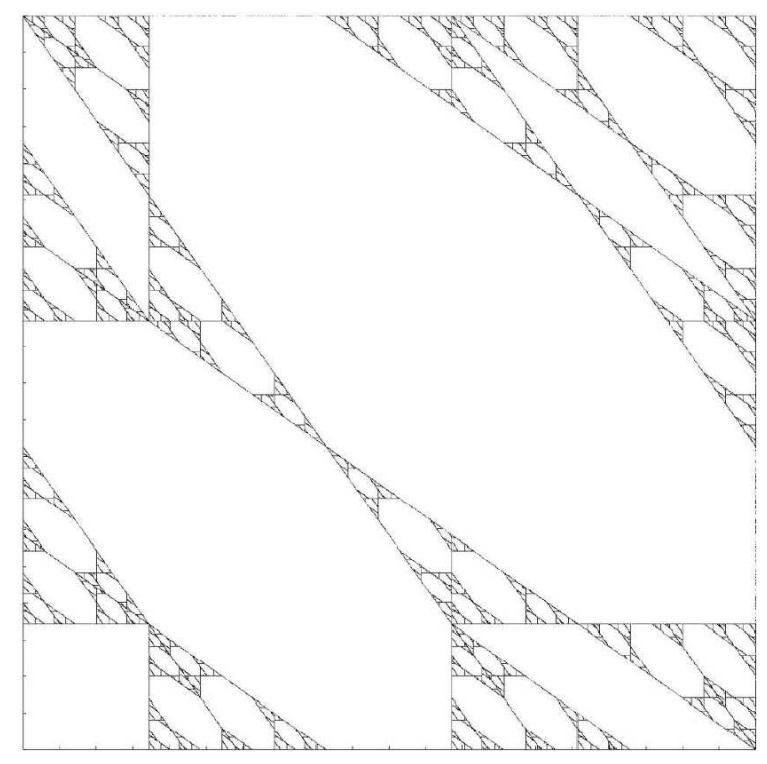

Figure 1: The phase space of a piecewise isometry of the torus. This plots shows the mosaic of cells of the map $K$ defined in equation (7), for the parameter $\lambda=\sqrt{2}$.

is known as pseudochaos ${ }^{1}$. We note that the chaos border has also been studied using probabilistic models $[2,31,32]$.

One model of pseudochaos can be derived from the kicked oscillator systems, which are the simplest and best studied maps for exploring the mechanism and transport properties of sticky orbits. This is also known as the stochastic web map, due to the presence, for al-

\footnotetext{
${ }^{1}$ This term has also a somewhat different usage [8], while other authors call it weak chaos [15].
} 
most all not too large parameter values, of a web-like stochastic layer within which chaotic orbits are typically unbounded [36]. Physically, the map describes a one-dimensional harmonic oscillator with position $x$ and momentum $y$ which is given an instantaneous kick in resonance with the natural oscillation frequency, with the amplitude of the kick equal to a periodic function $f(x)$. The map relating phase-space points preceding successive kicks is given by

$$
\left(\begin{array}{l}
x \\
y
\end{array}\right) \mapsto\left(\begin{array}{cc}
\cos \theta & \sin \theta \\
-\sin \theta & \cos \theta
\end{array}\right) \cdot\left(\begin{array}{c}
x \\
y+f(x)
\end{array}\right) \quad \theta=2 \pi r / s
$$

In the literature the kick function $f(x)$ is usually chosen to be $\lambda \sin (x)$ with $|\lambda|<2$, in which case numerical simulations reveal a stochastic web with apparent crystalline symmetry for $s=3,4,6$ and quasi-crystalline long-range order for $s=5,7,8, \ldots$. One has studied how the displacement of a phase point increases with "time" $t$, the number of iterations, and found asymptotic power-law behaviour $t^{\mu}$, where $\mu$ depends non-trivially on the parameter $\lambda$. For special values of $\lambda$ (found by numerical experiment), one finds sticky orbits associated with self-similar island structures.

The focus of the present investigation is a limiting case of the $s=4$ kicked-oscillator map, in which the kick function is, up to possible rescaling and a shift of origin, a discontinuous sawtooth function

$$
f(x)=\lambda(x \bmod 1) \quad \lambda=2 \cos (2 \pi p / q) .
$$

This model was introduced as an example of pseudochaos in [29].

With the choice $r / s=1 / 4$ and a simple change of variables, the map (1) becomes

$$
W(x, y)=(y,-x-f(y))
$$

and the periodicity of $f$ endows the map $W$ with a discrete translation symmetry with fundamental domain $\Omega=[0,1)^{2}$. Indeed, defining the $\pi / 2$ rotation

$$
F=\left(\begin{array}{cc}
0 & 1 \\
-1 & 0
\end{array}\right)
$$

then, clearly, for all $(m, n) \in \mathbb{Z}^{2}$

$$
W(x+m, y+n)=(y+n,-x-m-f(y))=W(x, y)+F \cdot(m, n)
$$

which implies at once that $W^{4}$ commutes with integer translations:

$$
W^{4}(x+m, y+n)=W^{4}(x, y)+(m, n) .
$$

The lattice translational symmetry gives us a decisive simplification as follows. Let us denote by $\lfloor\alpha\rfloor$ and by $\{\alpha\}=\alpha \bmod 1$, respectively, the integer part (floor) and fractional part of a real number $\alpha$, so that $\alpha=\{\alpha\}+\lfloor\alpha\rfloor$. Then, from (3) and (5)

$$
\begin{aligned}
W(x, y) & =W(\{x\}+\lfloor x\rfloor,\{y\}+\lfloor y\rfloor) \\
& =W(\{x\},\{y\})+F \cdot(\lfloor x\rfloor,\lfloor y\rfloor) \\
& =K(\{x\},\{y\})+(0,\lfloor-\{x\}-f(\{y\})\rfloor)+F \cdot(\lfloor x\rfloor,\lfloor y\rfloor)
\end{aligned}
$$


where

$$
K(a, b)=(b,\{-a-\lambda b)\}) \quad(a, b) \in \Omega .
$$

The action of $W$ on $\mathbb{R}^{2}$ is now decomposed into the action of $K$ on $\Omega$ and a lattice isometry. The former affects the latter but not vice-versa, and therefore we have a skew dynamical system on $\Omega \times \mathbb{Z}^{2}$. This decomposition will allow us to take advantage of the existing knowledge of the orbits of $K$ in order to describe and classify those of $W$ efficiently.

The piecewise affine map $K$ has been studied extensively since the late 1980's. Early interest was in its role as a second-order nonlinear digital filter, with the sequence $\left\lfloor v_{n}+\right.$ $\left.f\left(u_{n}\right)\right\rfloor, n=0,1, \ldots$, describing the overflow oscillations. A number of papers on digital filters $[4,9,10,12]$ explored the fractal structures generated by iteration of the map $K$. The implications of the map for dynamical systems theory became apparent when Ashwin showed [3] the equivalence of $K$ to the sawtooth standard map

$$
(x, y) \mapsto(x+f(x), y+x+f(x)),
$$

a linearized version of the standard map of Chirikov and Taylor, and obtained a number of general results regarding the periodic and aperiodic orbits, the latter generating fractal structures in the absence of true chaos (i.e., of non-zero Lyapunov exponent).

Perturbation of the sawtooth standard map by adding a small sinusoidal term to $f(x)$ has been shown by Dana [11] to produce a map which is conjugate to a kicked-oscillator map with a pure sinusoidal kick function. He has found and explained the mechanism for sticky orbits, within the stochastic web, which intermittently shadow accelerator modes and in this way escape to infinity with superdiffusive power-laws.

An additional role for $K$ was found in [25,28]. For certain rational rotation numbers $p / q$ in equation (2), the restriction of $K$ to a uniformly distributed, dense set of points of the unit square is conjugate to a map on the integer lattice $\mathbb{Z}^{2}$ describing elliptic motion on the plane, subject to round-off

$$
\tilde{K}:(x, y) \mapsto(\lfloor\tilde{\lambda} x\rfloor-y, x)
$$

where $\lambda$ and $\tilde{\lambda}$ are conjugate roots of a quadratic polynomial with integer coefficients. The dual mappings $K$ and $\tilde{K}$ generate fractal structures in the square and corresponding families of fractal "snowflake" orbits on the infinite lattice. In contrast to what we shall find for the maps $K$ and $W$, the fractal dimensions associated with the asymptotic longtime behaviour of the orbits of $K$ and $\tilde{K}$ are identical.

In recent years it has been recognized that the map $K$ on a square, recast as a piecewise rotation on a rhombus, provides a nice example of the general class of piecewise isometries $[1,5,6,16-22,24]$, generalizing to two dimensions the well studied notion of interval exchange maps. The case of quadratic irrational parameter $\lambda$ has been thoroughly studied, and we shall make extensive use of these results in the current work. The central theme of self-similarity in those models has been extended to a more general concept of recursive tiling in some cubic examples as well, where it has made possible a systematic analysis of periodic and aperiodic orbits $[20,26,27,33]$. 
As we have seen above, the sawtooth kicked-oscillator map $W$ decomposes into a local piecewise affine map $K$ (conjugate to a rotation by $2 \pi p / q=\cos ^{-1}(\lambda / 2)$ ) and a global lattice map (rotation by $\pi / 2$ followed by a lattice translation). Technically, it is useful to think of $W$ as lifting the well studied dynamics of the local map $K$ to the entire real plane. At that level, a new set of questions arises, such as whether orbits of $W$ lifted from periodic orbits of $K$ remain periodic, or become promoted to accelerator modes which escape ballistically to infinity. Similarly, one can ask whether the aperiodic orbits of $K$, confined to a fractal set of Lebesgue measure zero, are promoted to escape orbits, and if so, how rapidly (sub-diffusively, diffusively, or super-diffusively) the orbits move to infinity. After developing the necessary mathematical machinery, we shall answer these questions in a variety of examples with quadratic irrational $\lambda$.

Central to our analysis of the lifted map $W$ is the fact that the orbits of $W$ are governed by exactly the same symbolic dynamics as the orbits of $K$. To trace out the local orbit in $\Omega$, one associates an appropriate rotation and translation vector with each symbolic step. The accompanying lattice trajectory is obtained from the identical steps in symbol space, inserting global rotations and translation vectors in place of the local ones.

The asymptotic scaling behaviour of the $K$ and $W$-orbits is a consequence of the scaling of the respective local and global translation vectors. If successively finer length scales in $\Omega$ are assigned level numbers $L=0,1, \ldots$, then the local translations scale as $\omega^{L}$, $\omega<1$, whereas the corresponding iteration numbers scale as $\omega_{T}^{L}, \omega_{T}>1$. This gives rise to a fractal geometry of the aperiodic orbits ${ }^{2}$ of dimension $\log \omega_{T} / \log \omega$. As we shall see, there is a recursion relation for the global translation vectors as well, characterized by a matrix $M$. In some of the quadratic models, the recursion is trivial and the orbits remain within a uniformly bounded distance from the starting point. In other models, there is a unique largest eigenvalue of $M$, whose magnitude $\omega_{W}>1$ introduces a third important scale factor. In these models we have power-law growth, with asymptotic behaviour $t^{\mu}$, $\mu=\log \omega_{W} / \log \omega_{T}$. Whether the power-law is sub-diffusive, diffusive, super-diffusive, or ballistic therefore depends on the relative sizes of $\omega_{W}$ and $\omega_{T}$.

The remainder of the article will be organized as follows. We will begin, in section 2 , by stating and proving a number of general properties of 4-fold symmetric kicked-oscillator maps and their corresponding piecewise affine maps. After reviewing, in section 3, the conceptual and mathematical framework of recursive tiling in models with quadratic irrational parameter, we develop in section 4 some general criteria for power-law asymptotics. We then proceed to the details of the specific cases in section 5 , where we demonstrate rigorously the existence of orbits with various asymptotic behaviour, by verifying the above criteria using exact arithmetic of algebraic numbers. We conclude with a concise table of results for all of the models with quadratic $\lambda$ studied in [24].

\footnotetext{
${ }^{2}$ Unless stipulated otherwise, the term 'aperiodic' refers to the map $K$.
} 


\section{Basic properties}

We introduce the shorthand notation $z=(x, y),\{z\}=(\{x\},\{y\}),\lfloor z\rfloor=(\lfloor x\rfloor,\lfloor y\rfloor)$, and define the coding function $\iota$ as follows

$$
\iota(z)=\lfloor-\{x\}-\lambda\{y\}\rfloor=\iota(\{z\}) .
$$

The code $\left(\iota_{0}, \iota_{1}, \ldots\right)$ of the orbit of $z$ is defined by the equation $\iota_{k}=\iota\left(W^{k}(z)\right)$. (We shall use the symbol $\iota$ for both coding function and code, as this will not lead to confusion.) We now rewrite equation (6) as

$$
W(z)=K(\{z\})+(0, \iota(\{z\}))+F \cdot\lfloor z\rfloor .
$$

This equation can be iterated to give

Lemma 1 For all $n>0$ we have

$$
W^{n}(z)=K^{n}(\{z\})+\sum_{k=1}^{n} F^{n-k} \cdot\left(0, \iota_{k}\right)+F^{n} \cdot\lfloor z\rfloor .
$$

In particular, we have $\left\{W^{n}(z)\right\}=K^{n}(\{z\})$ and the escape rate is at most linear: $\left\|W^{n}(z)\right\|=$ $O(n)$ for $n \rightarrow \infty$.

Proof. We regard equation (8) as the base case for induction. Assuming the lemma to be valid up to $n$, then

$$
W^{n+1}(z)=W\left(K^{n}(\{z\})\right)+F \cdot\left(\sum_{k=1}^{n} F^{n-k} \cdot\left(0, \iota_{k}\right)+F^{n} \cdot\lfloor z\rfloor\right)
$$

as $K^{n}(\{z\})$ is the only non-integer quantity in the right-hand side of the equation. Thanks to $(8)$, we have

$$
\begin{aligned}
W^{n+1}(z)= & K^{n+1}(\{z\})+\left(0, \iota_{n}\right) \\
& +\sum_{k=1}^{n} F^{n+1-k} \cdot\left(0, \iota_{k}\right)+F^{n+1} \cdot\lfloor z\rfloor \\
= & K^{n+1}(\{z\})+\sum_{k=1}^{n+1} F^{n+1-k} \cdot\left(0, \iota_{k}\right)+F^{n+1} \cdot\lfloor z\rfloor
\end{aligned}
$$

which completes the proof of equation (9). The statement $\left\|W^{n}(z)\right\|=O(n)$ follows from the boundedness of the code, and the fact that the eigenvalues of $F$ belong to the unit circle. The validity of the equation $\left\{W^{n}(z)\right\}=K^{n}(\{z\})$ is evident.

Thus a periodic orbit for $W$ must give a periodic orbit for $K$ and an aperiodic orbit under $K$ must remain aperiodic when lifted to the plane. As to the converse, we have

Proposition 2 If $z \in \mathbb{R}^{2}$ is such that $\{z\}$ is periodic of period $T$ for $K$, then 
(i) If $\operatorname{gcd}(T, 4)=1$, then $z$ is periodic for $W$, and its period is one of $T, 2 T, 4 T$.

(ii) If $\operatorname{gcd}(T, 4)=2$, then $z$ is periodic for $W$, and its period is one of $T, 2 T$.

(iii) If $\operatorname{gcd}(T, 4)=4$, then $W^{T}$ acts as a translation

$$
W^{k T}(z)=z+k v
$$

where $v$ is an integer vector such that $\|v\| \leq T\lfloor 1+|\lambda|\rfloor$.

Notice that $v$ may be zero, and in this case $z$ is a $T$-periodic point for $W$.

Proof. Let $z$ be such that $\{z\}$ is a periodic point of period $T$ for $K$, and let $\left(\iota_{0}, \iota_{1}, \cdots\right)$ be its code. Assume that $T$ is coprime to 4 . Then, for any integer $a$, the integers $a, a+$ $T, a+2 T, a+3 T$ are congruent modulo 4 to $0,1,2,3$, in some order. From equation (9), and recalling that $F^{4}=I d$, we obtain

$$
\begin{aligned}
W^{4 T}(z) & =K^{4 T}(\{z\})+\sum_{k=1}^{4 T} F^{4 T-k} \cdot\left(0, \iota_{k}\right)+F^{4 T} \cdot\lfloor z\rfloor \\
& =\{z\}+\lfloor z\rfloor+\sum_{k=1}^{T}\left(F^{-k}+F^{-k+T}+F^{-k+2 T}+F^{-k+3 T}\right) \cdot\left(0, \iota_{k}\right) \\
& =z+\sum_{k=1}^{T}\left(F^{0}+F^{1}+F^{2}+F^{3}\right) \cdot\left(0, \iota_{k}\right)=z .
\end{aligned}
$$

If $T \equiv 2(\bmod 4)$, then $2 T$ is a multiple of 4 , and hence

$$
\begin{aligned}
W^{2 T}(z) & =\{z\}+\lfloor z\rfloor+\sum_{k=1}^{T}\left(F^{-k}+F^{-k+T}\right) \cdot\left(0, \iota_{k}\right) \\
& =z+\sum_{k=1}^{T}\left(F^{-k}+F^{-k+2}\right) \cdot\left(0, \iota_{k}\right) \\
& =z+\sum_{k=1}^{T}\left(F^{-k}-F^{-k}\right) \cdot\left(0, \iota_{k}\right)=z .
\end{aligned}
$$

Finally, if $T \equiv 0(\bmod 4)$, we have that

$$
W^{T}(z)=z+\sum_{k=1}^{T} F^{-k} \cdot\left(0, \iota_{k}\right)=z+(a, b)
$$

where

$$
a=\sum_{k=0}^{T / 4-1}\left(\iota_{4 k+3}-\iota_{4 k+1}\right) \quad b=\sum_{k=0}^{T / 4-1}\left(\iota_{4 k+4}-\iota_{4 k+2}\right) .
$$

Thus

$$
W^{j T} z=z+j v
$$




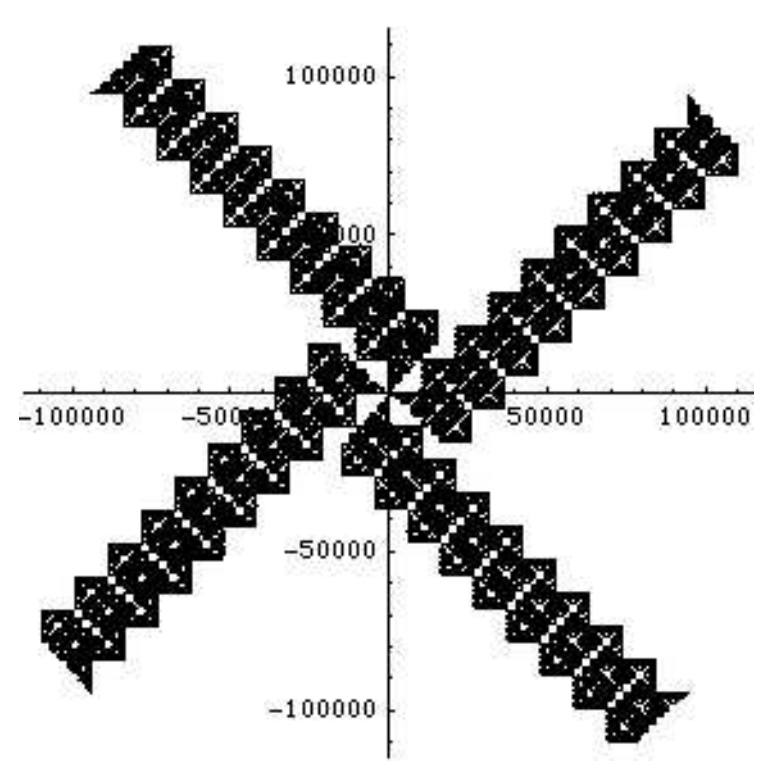

Figure 2: Accelerator mode for kicked-oscillator map $W$ with $\lambda=-\sqrt{3}$. The local rotation period is $T=1182611492$, and the displacement per period is $v=(10496,-10496)$.

where

$$
\|v\|=\left\|\sum_{k=1}^{T} F^{T-k} \cdot\left(0, \iota_{k}\right)\right\| \leq T \max \left|\iota_{k}\right| \leq T\lfloor 1+|\lambda|\rfloor .
$$

We see from proposition 2 that it is possible for some periodic orbits of $K$ to be lifted to accelerator modes, for which the orbits of $W^{4}$ move to infinity with constant average velocities. Figure 2 shows a long-period accelerator mode for $\lambda=-\sqrt{3}$. When both periodic and accelerator-mode orbits coexist in a model, there may also be hybrid aperiodic orbits which spend part of the time shadowing periodic orbits and part shadowing accelerator modes, the net effect being power-law asymptotic behaviour with a power strictly less than unity. Similar sticky orbits were studied by Dana [11] in the context of the sine kicked-oscillator map. In our models, all aperiodic orbits are sticky, and so we might expect that in those cases where accelerator-mode and periodic orbits coexist, typical aperiodic orbits will exhibit power-law long-time behaviour. We will present several such examples in section 5 .

\section{Self-similarity in quadratic models}

In this section we review the role of self-similarity in organizing the orbits of the map $K$ on the square $\Omega$, and then extend the formalism to the map $W$ on the plane. We shall see that the same hierarchy of polygonal return-map domains, with its associated symbolic representation, serves as the organizing principle for both cases. In order to make immediate use of the results of [24], we will need to generalize slightly the definition of $K$, 
introducing an additional non-zero real parameter $\tau$. The map is defined on the half-open square $\Omega=[0, \tau)^{2}$ for $\tau>0$ and $(\tau, 0]^{2}$ for $\tau<0$. Introducing $\tau$-rescaled brackets

$$
\{x\}_{\tau} \stackrel{\text { def }}{=}\{x / \tau\} \tau \quad\lfloor x\rfloor_{\tau} \stackrel{\text { def }}{=}\lfloor x / \tau\rfloor \tau,
$$

we now define

$$
\begin{gathered}
f(x) \stackrel{\text { def }}{=} \lambda\{x\}_{\tau} \quad x \in \mathbb{R} \\
K(u, v)=\left(v,\{-u-f(v)\}_{\tau}\right) \quad(u, v) \in \Omega .
\end{gathered}
$$

With the redefined function $f$, the defining relation (3) for $W$ remains valid. It can be verified that the statements and proofs of lemma 1 and proposition 2 remain valid if all fractional parts and floors are replaced by their $\tau$-rescaled counterparts. For the sake of algebraic simplicity, we follow [24] and take $\tau$ to be the negative of $\tilde{\lambda}^{-1}$, where $\lambda$ and $\tilde{\lambda}$ are conjugate solutions of the same quadratic equation. Specifically, $\tau=1 / \lambda$ for $\lambda= \pm \sqrt{2}, \pm \sqrt{3}$, and $\tau=\lambda$ for the other examples.

\subsection{Recursion for local orbits}

The map $K$ partitions the square $\Omega$ into a small number of polygonal domains $\Omega_{j}$, on each of which it acts as a linear transformation

$$
C=\left(\begin{array}{cc}
0 & 1 \\
-1 & -\lambda
\end{array}\right) \quad|\lambda|<2
$$

followed by a $j$-dependent translation by an integer multiple of $(0, \tau)$. An example is shown in figure 3 .

Geometrical self-similarity takes the form of a nested sequence of similar triangles (see figures 4) $\mathcal{D}(L), L=0,1, \ldots$ converging to a point $u_{\infty}$, and related to one another by a uniform scale transformation

$$
\mathcal{D}(L+1)=\omega \mathcal{D}(L)
$$

which is performed with respect to the point $u_{\infty}$, via the real scaling constant $\omega \in(0,1)$.

Dynamical self-similarity requires that the level- $(L+1)$ first-return map $\rho(L+1)$ is just the rescaled version of $\rho(L)$ (again in coordinates relative to $u_{\infty}$ )

$$
\rho(L+1)=\omega \circ \rho(L) \circ \omega^{-1} .
$$

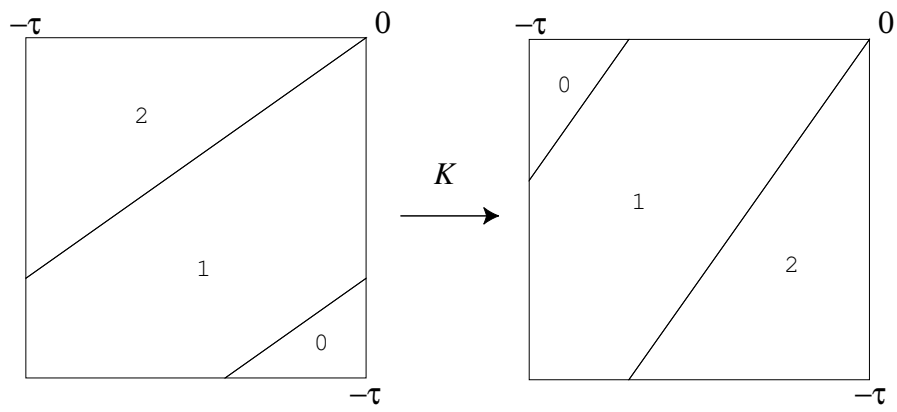

Figure 3: The piecewise affine map $K$ acting on the square $\Omega=(\tau, 0]^{2}$, for $\lambda=-\sqrt{2}=2 \tau$. 
In addition, associated to the return map $\rho(L)$ there exists a partition of $\mathcal{D}(L)$ into subdomains $\mathcal{D}_{j}(L), j \in I_{J} \stackrel{\text { def }}{=}\{0, \ldots, J-1\}$, with the property that, for each $j$, there exists an $L$-independent return path

$$
p(j)=\left(p(j, 0), p(j, 1), \ldots, p\left(j, \nu_{j}-1\right)\right)
$$

such that for any $L \geq 0$,

$$
\rho_{j}(L+1)=\rho_{p\left(j, \nu_{j}-1\right)}(L) \circ \cdots \circ \rho_{p(j, 1)}(L) \circ \rho_{p(j, 0)}(L),
$$

where $\rho_{j}(L)$ is $\rho(L)$ restricted to $\mathcal{D}_{j}(L)$. Thus the integer $p(j, k)$ is the index of the subdomain of $\mathcal{D}(L)$ that $\mathcal{D}(L+1)$ visits at the $k$-th iterate of the return map $\rho(L)$. The return orbits of $\mathcal{D}_{j}(1)$ in $\mathcal{D}(0)$ are illustrated in figure 5 a.

The function $p$ appearing in the above formulae is called the path function. It is assumed that $\mathcal{D}(L+1) \subset \mathcal{D}_{k}(L)$ for some $k$, so that $p(j, 0)=k$ for all $j$. The level- $L$ first-return time $T_{j}(L)$ associated with $\rho_{j}(L)$ is conveniently calculated with the aid of the incidence matrix $A$ defined by

$$
A_{j i}=\#\{k: p(j, k)=i\} \quad i, j \in I_{J} .
$$

Specifically,

$$
T_{j}(L)=\sum_{k=0}^{\nu_{j}-1} T_{p(j, k)}(L-1)=\sum_{i=0}^{J-1} A_{j i} T_{i}(L-1) .
$$

With this linear recursion relation, it is easy to establish criteria for the $\omega_{T}^{L}$ scaling of the level- $L$ return times $T_{j}(L)$.

Lemma 3 Let $A$ be the incidence matrix (13) associated with the scaling sequence $\mathcal{D}(L)$, $L=0,1, \ldots$ Suppose that $A$ has a largest eigenvalue $\omega_{T}>1$ which is unique and isolated, and that $\sum_{j} \pi_{i j} T_{j}(0)>0, i \in I_{J}$, where $\pi$ is the projection matrix associated with this eigenvalue. Then the quantities $T_{j}(L) \omega_{T}^{-L}$ tend to positive limits for $L \rightarrow \infty$.

Note that the assumptions of the lemma are verified if $A^{n}$ is a positive matrix for some $n \in \mathbb{N}$, from Perron-Frobenius theorem. Also, the local spatial scale factor $\omega$ and the temporal scale factor $\omega_{T}$ together determine the Hausdorff dimension ${ }^{3}$ of the set of aperiodic orbits [27].

Proof. In matrix notation, (14) becomes

$$
T(L)=A^{L}(\pi T(0)+(1-\pi) T(0)),
$$

and the asymptotic estimate for $T_{j}(L)$ follows, with $B_{j}=\sum_{j^{\prime}} \pi_{j j^{\prime}} T_{j^{\prime}}(0)>0$.

At the lowest level of the scaling hierarchy are the domains $\mathcal{D}_{j}(0)$, whose orbits return to $\mathcal{D}(0)$ after $\nu_{0 j} \stackrel{\text { def }}{=} T_{j}(0)$ iterations of $K$, along paths specified by a function $p_{0}(j, t)$ 


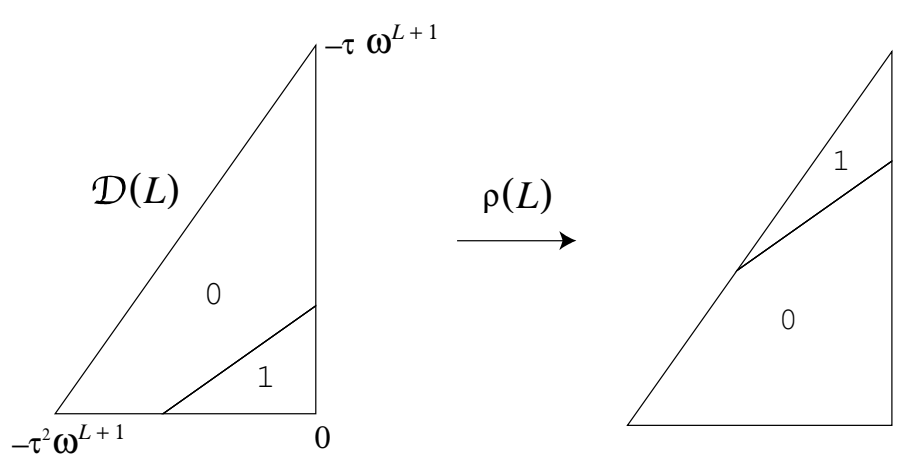

Figure 4: Return map of scaling triangle $\mathcal{D}(L)$, for $\lambda=-\sqrt{2}$. Here the origin of coordinates has been shifted to the scaling limit point $u_{\infty}$ at the lower right-hand vertex. The nested triangles scale according to (11), with $\omega=3-2 \sqrt{2}$.

(see figure 5a)). The return times $T_{j}(0)$ are essential to initiate the temporal recursion relation (14), but the explicit return paths through $\Omega$ are of little interest, since they are not repeated in higher levels. In contrast, the path function $p(j, t)$ applies to every level- $L$ to level- $(L+1)$ transition.

In reference [24] it was shown that for all of the cases with quadratic $\lambda$ and domain ${ }^{4}$ $\Omega$ there are either one or (in the case $\lambda=-\sqrt{3}$ ) two scaling sequences of nested domains, and that the periodic domains associated with such scaling sequences completely tile $\Omega$, up to a finite number of periodic domains which do not scale, and a set of Lebesgue measure zero. A complementary tiling result was established in [27]: for any level $L$, the orbits of the return-map sub-domains $\mathcal{D}_{j}(L)$ (for $\lambda=-\sqrt{3}$ this includes both scaling sequences) tile $\Omega$, up to a finite number of periodic domains and a set of measure zero. For $L \rightarrow \infty$, the orbits of the $\mathcal{D}_{j}(L)$ form a sequence of successively finer covering sets for the discontinuity-avoiding aperiodic orbits (residual set). The covering of the residual set by a high-level return map is illustrated in figure $5 \mathrm{c}$.

The recursive tiling property has been extended in [27] to include cases where $\lambda$ is not a quadratic irrational.

We define a tile of level $L$ by

$$
\mathcal{D}_{j}^{\mathbf{t}}(L) \stackrel{\text { def }}{=} K^{t_{0}} \circ \rho^{t_{1}}(0) \circ \rho^{t_{2}}(1) \circ \cdots \circ \rho^{t_{L}}(L-1) \mathcal{D}_{j}(L)
$$

where $\mathbf{t}=\left(t_{0}, t_{1}, \ldots, t_{L}\right)$ and $\rho^{t}(L)$ is the $t$-th iterate of $\rho(L)$. The level- $L$ union of returnmap orbits of a particular scaling sequence is just the set $\left\{\mathcal{D}_{j}^{\mathrm{t}}(L): j \in I_{J}, t_{i}=0, \ldots, \nu_{i}-1\right.$, $i=0, \ldots, L\}$.

To each tile we associate a symbolic representation encoding the recursive history of its construction. Specifically, the tile $\mathcal{D}_{j}^{\mathbf{t}}(L)$ will be coded by the finite sequence of integer

\footnotetext{
${ }^{3}$ Due to exact self-similarity, the Hausdorff dimension coincides with the box-counting dimension.

${ }^{4}$ These results refer to the specific cases with $\Omega=[0, \tau)^{2}$ (positive $\lambda$ ) or $\Omega=(\tau, 0]^{2}$ (negative $\lambda$ ); changing fundamental domain changes the dynamics, in general.
} 


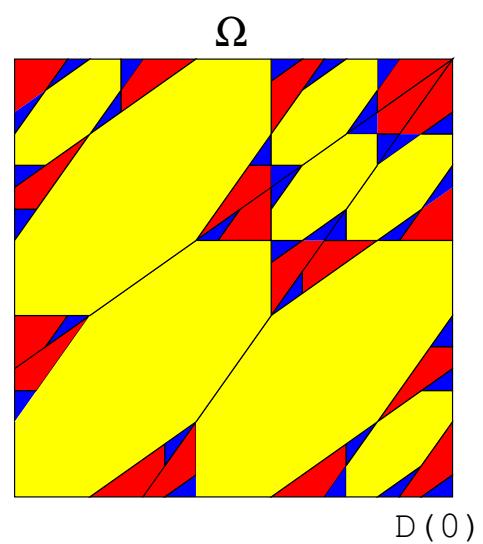

(a)

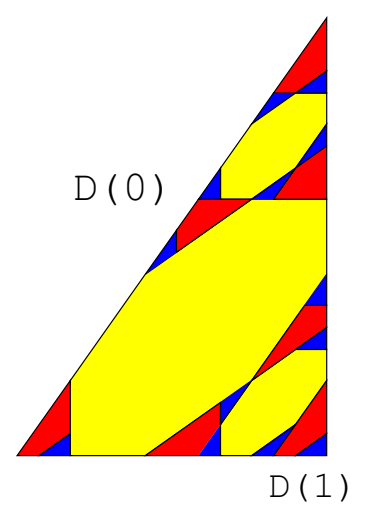

(b)

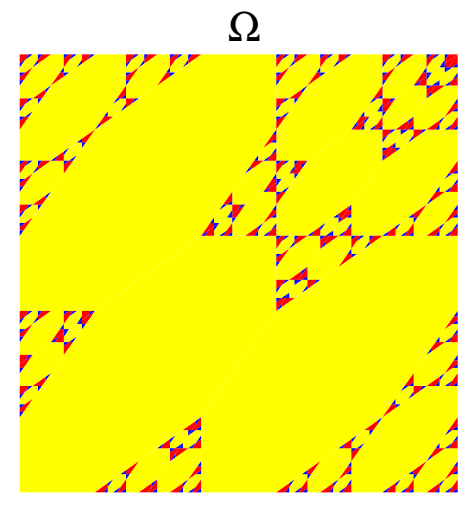

$\mathrm{D}(1)$

(c)

Figure 5: (a) Return orbits of $\mathcal{D}_{0}(0)$ (red) and $\mathcal{D}_{1}(0)$ (blue), for $\lambda=-\sqrt{2}$. After $\nu_{00}=19$ iterations of $K$, the orbit of $\mathcal{D}_{0}(0)$ returns to $\mathcal{D}(0)$; after $\nu_{01}=34$ iterations of $K$, the orbit of $\mathcal{D}_{1}(0)$ also returns to $\mathcal{D}(0)$. The net effect is the return map of $\mathcal{D}(0)$ displayed in figure 4 . (b) Return orbits, within $\mathcal{D}(0)$, of $\mathcal{D}_{0}(1)$ and $\mathcal{D}_{1}(1)$. After $\nu_{0}=7$ (resp. $\nu_{1}=13$ ) iterations of $\rho(0)$, $\mathcal{D}_{0}(1)$ (resp. $\mathcal{D}_{1}(1)$ ) returns to $\mathcal{D}(1)$. The net effect is the return map $\rho(1)$, the rescaled version of $\rho(0)$. (c) Return orbits, within $\Omega$, of $\mathcal{D}_{0}(1)$ (red) and $\mathcal{D}_{1}(1)$ (blue). Respective return times are $T_{0}(1)=163$ and $T_{1}(1)=322$. These orbits tile a region complementary to the largest periodic domains (octagons), and cover all the discontinuity-avoiding aperiodic orbits (residual set) of $K$.

pairs

$$
\left(\left(j_{0}, t_{0}\right),\left(j_{1}, t_{1}\right), \ldots,\left(j_{L}, t_{L}\right)\right)
$$

with $j_{L}=j$ and all other $j_{i}$ uniquely determined by the path functions

$$
j_{0}=p_{0}\left(j_{1}, t_{1}\right) \quad j_{i}=p\left(j_{i+1}, t_{i+1}\right), \quad i>0 .
$$

The above equation shows that such a geometric code contains redundant information; nonetheless, this coding turns out to be more convenient than the dynamical coding $\iota$ for describing the scaling phenomena which are relevant here.

A point of the residual set (the complement of the discontinuity set in its closure) lies in the intersection of a countable sequence of nested tiles, and can thus be specified by an infinite symbol sequence

$$
\left(\left(j_{0}, t_{0}\right),\left(j_{1}, t_{1}\right), \ldots\right)
$$

That the residual set is nonempty is guaranteed by certain sufficient conditions $[27,33]$. In particular, one can prove that the Hausdorff dimension of the set is nontrivial, and that the Hausdorff measure is positive (for background, see [14]).

The action of the map $K$ is expressed symbolically as follows [26,33]: if $i$ is the smallest index $k$ such that $t_{k}$ is less than its maximum possible value $\nu_{j_{k}}-1$, then

$$
\left(\left(j_{0}, t_{0}\right),\left(j_{1}, t_{1}\right), \ldots,\left(j_{i}, t_{i}\right), \ldots\right) \mapsto\left(\left(j_{0}^{\prime}, 0\right),\left(j_{1}^{\prime}, 0\right), \ldots,\left(j_{i-1}^{\prime}, 0\right),\left(j_{i}, t_{i}+1\right),\left(j_{i+1}, t_{i+1}\right), \ldots\right),
$$

where the $j_{k}^{\prime}$ are determined uniquely by the constraints (15). In the special case where all $t_{k}$ are maximal, the successor sequence has all members $\left(j_{k}^{\prime}, 0\right)$ determined by (15) (recall $p(j, 0)$ and $p_{0}(j, 0)$ are independent of $\left.j\right)$; in particular, such sequence is unique. This is 
an instance of a Vershik map, which can be likened to the action of time on a calendar, whereby a hierarchy of clocks with periodicities $\nu_{i}$ (hour, day, month, etc.) are increased and periodically reset. Under certain conditions which are satisfied in the presence of scaling domains, the Vershik map is uniquely ergodic on an appropriate graph [33].

\subsection{Recursion for global orbits}

We now represent the euclidean plane $\mathbb{R}^{2}$ as $\Omega \times \mathbb{Z}[i]$ where

$$
\mathbb{Z}[i]=\left\{m_{1}+m_{2} i: m_{1,2} \in \mathbb{Z}, i=\sqrt{-1}\right\}
$$

is the ring of Gaussian integers. This is done in two stages. First, we let the map $W$ act on the pair $[u, m] \in \Omega \times \mathbb{Z}^{2}$ as

$$
W[u, m]=[K(u), F \cdot m+(0, \Delta(u))]
$$

where $F$ was defined in (4) and for $(x, y) \in \Omega$,

$$
\Delta(x, y)=\left\lfloor-\tau^{-1}(x+\lambda y)\right\rfloor .
$$

Then we identify $\mathbb{Z}^{2}$ with $\mathbb{Z}[i]$ via

$$
\left(m_{1}, m_{2}\right) \longleftrightarrow \zeta=m_{2}+m_{1} i
$$

and one verifies that the action of $F$ becomes multiplication by $i$. The action of $W$ is thus

$$
W[u, \zeta]=[K(u), i \zeta+\Delta(u)] \quad[u, \zeta] \in \Omega \times \mathbb{Z}[i] .
$$

To reach level 0 of the scaling hierarchy, we use lemma 1 to obtain

$$
\sigma(0)\left[\mathcal{D}_{j}(0), \zeta\right] \stackrel{\text { def }}{=} W^{T_{j}(0)}\left[D_{j}(0), \zeta\right]=\left[\rho(0) \mathcal{D}_{j}(0), i^{T_{j}(0)} \zeta+d_{j}(0)\right]
$$

where

$$
d_{j}(0)=\sum_{t=1}^{T_{j}(0)-1} i^{T_{j}(0)-t} \Delta\left(K^{t} \mathcal{D}_{j}(0)\right) .
$$

After $T_{j}(0)$ iterations, the image of a point in $\left[\mathcal{D}_{j}(0), \zeta\right]$ is not necessarily in the lattice cell labeled by $\zeta$; that is, we do not necessarily have a true return. What is true, however, is that during the $T_{j}(0)$ iterations, there is no branching: the mapped domain remains intact, not intersecting any cell boundary ( $x$ or $y$ an integer multiple of $\tau$ ). This means we can still use the lifts of the return orbits on $\Omega$ as building blocks for finite or infinite orbits of $W$ on the plane. An example of the $W$-orbits of $\left[\mathcal{D}_{j}(0), 0\right], j \in I_{J}$ is shown in figure 6 .

We obtain the level- $L$ analogue of (18) by lifting the respective return paths of $\rho(L)$. We define, for any non-negative integer $L$ and $u \in \mathcal{D}_{j}(L), \zeta \in \mathbb{Z}[i]$,

$$
\sigma(L)[u, \zeta] \stackrel{\text { def }}{=} W^{T_{j}(L)}[u, \zeta]
$$




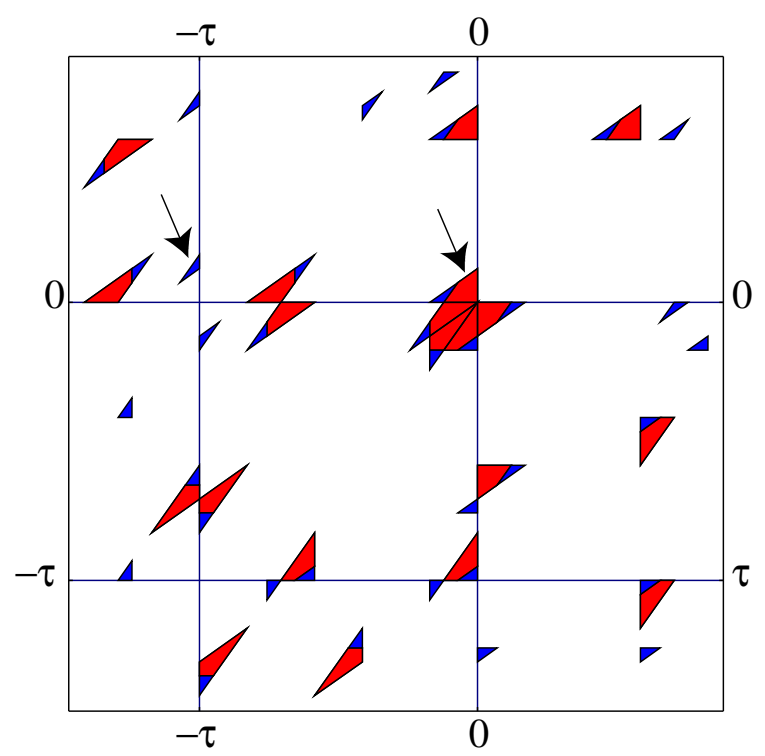

Figure 6: The $W$-orbits of $\left[\mathcal{D}_{0}(0), 0\right]($ red $)$ and $\left[\mathcal{D}_{1}(0), 0\right]$ (blue) for $\lambda=-\sqrt{2}$. The return times and symbolic paths are the same as those of the $K$-orbits of $\mathcal{D}_{0}(0)$ and $\mathcal{D}_{1}(0)$ shown in figure $5(\mathrm{a})$. Since the orbits terminate in domains (indicated by arrows) in cells labeled by Gaussian integers -1 and $-1+i$, we set $d_{0}(0)$ and $d_{1}(0)$ equal to those values. This method was applied repeatedly in the examples of section 5 .

where, by lemma 1, the right-hand side takes the form

$$
\left[\rho(L) u, i^{T_{j}(L)} \zeta+d_{j}(L)\right]
$$

The level- $L$ displacement $d_{j}(L)$ can be evaluated as in (18), but it is more useful to apply the recursion relation

$$
\sigma(L+1)=\sigma_{p\left(j, \nu_{j}-1\right)}(L) \circ \cdots \circ \sigma_{p(j, 0)}(L)
$$

to obtain a recursive formula.

Proposition 4 If $T_{j}(L) \equiv T_{j}(0)(\bmod 4)$ for $L=1,2, \ldots$, then we have the recursion relation

$$
d_{j}(L+1)=\sum_{k=0}^{J} c_{k}(p(j)) d_{k}(L),
$$

where $p(j)$ was defined in (12), and where for any sequence $a=\left(a_{0}, a_{1}, \ldots, a_{\nu-1}\right)$ of elements of $I_{J}$, we define

$$
T(a) \equiv \sum_{s=0}^{\nu-1} T_{a_{s}}(0)(\bmod 4) \quad c_{j}(a)=\sum_{s=0}^{\nu-1} \delta_{j, a_{s}} i^{T\left(a_{s+1}, \ldots, a_{\nu-1}\right)},
$$

with $\delta_{j, k}$ the Kronecker symbol.

We remark that $T(a)$ is independent of $L$. 
Proof. Substituting (19) into (20), we have, for all $u \in \mathcal{D}_{j}(L+1), \zeta \in \mathbb{Z}[i]$,

$$
\sigma(L+1)[u, \zeta]=\left[\rho(L+1) u, i^{T(p(j))} \zeta+d_{j}(L+1)\right]
$$

where

$$
d_{j}(L+1)=\sum_{t=0}^{\nu_{j}-1} i^{T(p(j \mid t+1))} d_{p(j \mid t)}(L),
$$

with the notation $p(j \mid t)$ representing the partial path starting at $t$,

$$
p(j \mid t)=\left(p(j, t), p(j, t+1), \ldots, p\left(j, \nu_{j}-1\right)\right) .
$$

The result follows by combining terms with the same value of $p(j, t)$.

In matrix notation, we write $d_{j}(L)$ as the $j$ th component of a of vector in $\mathbb{Z}[i]^{J}$, so that the recursion relation of proposition 4 takes the concise form

$$
d(L+1)=M \cdot d(L)
$$

with

$$
M_{j k}=c_{k}(p(j)), \quad j, k \in I_{J}
$$

The assumption concerning the $L$-independence, modulo 4 , of $T_{j}(L)$ is clearly indispensable to having a useful global recursion scheme. While at first sight this assumption may seem overly restrictive, it is in fact satisfied in all of the models we have studied (see section 4 below).

As we shall soon see, the properties of the matrix $M$ are of crucial importance for the asymptotics of aperiodic orbits. Useful in this regard is the Jordan canonical decomposition

$$
M=S \mathcal{M} S^{-1},
$$

where $\mathcal{M}$ is upper-triangular and $S$ is invertible. In specific models, we find that in some cases the diagonal elements $\mathcal{M}_{j, j}$ are all zeros and ones, while in the others there is a unique largest diagonal element, say, $\mathcal{M}_{J-1, J-1} \stackrel{\text { def }}{=} \omega_{W}>1$. In the former case, the aperiodic orbits are either bounded or tend to infinity logarithmically in the asymptotic long-time limit, while in the latter case the orbits are unbounded, with asymptotic powerlaw behaviour. In such a model, the quantity $\omega_{W}$ serves as a global geometric scale factor which, together with the temporal factor $\omega_{T}$, determines the exponent $\mu$ of the power law.

In the next section, we shall prove some general results regarding power-law asymptotics in our models, assuming throughout that the global recursion matrix has a unique largest eigenvalue $\omega_{W}>1$. The uniqueness assumption is probably stronger than necessary, but simplifies the treatment significantly and is sufficient to handle all of the known examples. Asymptotically, for $L \rightarrow \infty$, we have for the $L$ th power of $M$,

$$
\left(M^{L}\right)_{i, j}=S_{i, J-1} \omega_{W}^{L} S_{J-1, j}^{-1}+O\left(\omega^{L}\right)
$$


where $\omega^{\prime}<\omega_{W}$. Thus, the array of displacements $d(L)$ scales asymptotically as

$$
d(L)=M^{L} \cdot d(0)=\omega_{W}^{L} \xi+O\left(\omega^{L}\right),
$$

where

$$
\xi_{j}=\sum_{i \in I_{J}} S_{j, J-1} S_{J-1, i}^{-1} d_{i}(0)
$$

This $J$-dimensional vector will play an important role in formulating sufficient conditions for asymptotic $\omega_{W}^{L}$ scaling.

\section{Asymptotic power-law behaviour}

Power-law long-time behaviour of orbits is associated with global self-similarity in many kicked-oscillator models. Thus far we have investigated only those sawtooth oscillator models with a quadratic irrational parameter $\lambda=2 \cos 2 \pi p / q, p, q \in \mathbb{Z}$, but we see no reason why the results of this section should not apply equally well to non-quadratic cases where self-similarity is present (for example the cubic example of [27]). Self-similarity in these models refers to a specific scaling sequence $\mathcal{D}=\{\mathcal{D}(L): L=0,1, \ldots\}$, and the aperiodic orbits which belong to $\mathcal{D}$. Each point on such an orbit lies in a countable intersection of nested tiles of $\mathcal{D}$, encoded by a symbolic sequence of the form $\left(\left(j_{0}, t_{0}\right),\left(j_{1}, t_{1}\right), \ldots\right)$. For such an orbit, the long-time behaviour will depend in large part on the properties of the global recursion matrix $M$ associated with $\mathcal{D}$. The key property is what we will call global expansivity.

Definition 1 A scaling sequence is called globally expansive if the corresponding return times $T_{j}(L)$ satisfy the hypothesis of proposition 4, and if the incidence matrix $A$ (13) and global recursion matrix $M$ (23) have non-degenerate largest-magnitude eigenvalues $\omega_{T}>1$ and $\omega_{W}>1$, respectively.

In all quadratic models we have studied, the congruence relation on return times required by proposition 4 was found always to hold, and so was the spectral condition on the incidence matrix $A$. By constrast, the spectral condition on the global recursion matrix $M$ was found to hold in about one-half of the cases (see table 1).

We now turn to the question of under what circumstances and in what form the forward orbit $O_{+}(z)=\left\{W^{s} z: s=0,1, \ldots\right\}$ of a point belonging to a globally expansive scaling sequence will exhibit non-trivial long-time power-law behaviour. From numerical explorations we do not expect a uniform expansion of the orbit, since there are numerous examples of recurrence, i.e., the return of a long orbit, infinitely many times, to a neighborhood of its starting point. Rather we expect the power law to characterize what we shall call a rapid-ascent sub-orbit, defined as follows:

Definition 2 Let $z$ be an aperiodic point belonging to a globally expansive scaling sequence D. A rapid-ascent sub-orbit of the forward $W$-orbit $O_{+}(z)$, is a sub-orbit $\left(z_{0}, z_{1}, \ldots\right)$, 
$z_{k}=\left[u_{k}, \zeta_{k}\right]$, with $u_{k} \in \mathcal{D}\left(L_{k}\right)$, where $L_{0}, L_{1}, \ldots$ are monotonically increasing non-negative integers.

We now state our first main result, using the notation of section 3 .

Proposition 5 Let $z=[u, \zeta]$ be an aperiodic point belonging to a globally expansive scaling sequence $\mathcal{D}$ with symbolic representation $\left(\left(j_{0}, t_{0}\right),\left(j_{1}, t_{1}\right), \ldots\right)$. Suppose that for every $L>0$ such that $t_{L}$ is not maximal,

$$
\xi \cdot c\left(\left(p\left(j_{L} \mid t_{L}+1\right) \neq 0 .\right.\right.
$$

Then $O_{+}(z)$ contains a rapid-ascent sub-orbit $\left(z_{0}, z_{1}, \ldots\right), z_{k}=\left[u_{k}, \zeta_{k}\right]$, with $u_{k} \in \mathcal{D}\left(L_{k}\right)$, satisfying the following properties:

(i) Temporal scaling. Define the $k$ th iteration number $s_{k}$ by $z_{k}=W^{s_{k}} z$. There exist positive constants $\beta_{ \pm}$such that for all positive $k$,

$$
\beta_{-} \omega_{T}^{L_{k-1}}<s_{k}<\beta_{+} \omega_{T}^{L_{k-1}} .
$$

(ii) Local power law. For all $k$, we have

$$
\left\|u_{k}-u_{\infty}\right\| \leq|\mathcal{D}(0)| \omega^{L_{k}} .
$$

Moreover, there exists a positive $\eta$ such that

$$
\left\|u_{k}-u_{\infty}\right\| \leq \eta s_{k}^{-\nu}, \quad \nu=\left|\frac{\log \omega}{\log \omega_{T}}\right| .
$$

(iii) Global power law. We have

$$
0<\overline{\lim }_{k \rightarrow \infty} \frac{\left|\zeta_{k}\right|}{\omega_{W}^{L_{k-1}}}<\infty
$$

Moreover, there exists a positive $\mu$ such that

$$
0<\overline{\lim }_{k \rightarrow \infty} \frac{\left|\zeta_{k}\right|}{s_{k}^{\mu},}<\infty, \quad \mu=\frac{\log \omega_{W}}{\log \omega_{T}}
$$

The proof of proposition 5 is presented in Appendix A.

The condition (25) is not difficult to check in particular examples, since there are only a few distinct partial return paths $p(j \mid t)$ in the range $1 \leq t \leq \nu_{j}-1$. (Note that $t=0$ is excluded by the recursion relation for $z_{L}$ : if the $(L+1)$ st symbol is of the form $\left(j_{L+1}^{\prime}, 0\right)$, so that $u_{L} \in \mathcal{D}(L+1)$, the recursion rule sets $z_{L+1}=z_{L}$.) If we can verify the non-vanishing of $c(p(j \mid t)) \cdot \xi$ for all of them, we are done. This strategy will be extremely useful in the examples of the next section.

All of the specific examples of aperiodic points we shall discuss in section 5 have eventually periodic symbolic sequences. In such cases we can obtain a rather detailed description of the asymptotic behaviour: with rare exceptions, each point of the forward $\sigma(0)$ orbit of an aperiodic point in the scaling domain $[\mathcal{D}(0), \zeta]$, from some point onward, initiates a rapid-ascent sub-orbit with local and global power-law behaviour. 
Proposition 6 Let $z=[u, \zeta]$, with $u \in \mathcal{D}(0)$ an aperiodic point belonging to a globally expansive scaling sequence $\mathcal{D}$. Let $\left(\left(j_{0}, 0\right),\left(j_{1}, t_{1}\right), \ldots\right)$ be the symbolic representation of $z$, and suppose that $\left(j_{L}, t_{L}\right)=\left(j_{L+P}, t_{L+P}\right)$ for some positive $P$ and all sufficiently large $L$. Then there exists $s \geq 0$ such that every $z_{0}=W^{s_{0}} z \in \mathcal{D}(0), s_{0} \geq s$, initiates a rapid-ascent sub-orbit $\left(z_{0}, z_{1}, \ldots\right), z_{k}=\left[u_{k}, \zeta_{k}\right]=W^{s_{k}} z$, with $u_{k} \in \mathcal{D}(k P)$ such that the following quantities have finite $k \rightarrow \infty$ limits:

$$
\frac{s_{k}}{\omega_{T}^{k P}} \quad \frac{u-u_{\infty}}{\omega^{k P}} \quad \frac{u-u_{\infty}}{s_{k}^{-\nu}}
$$

with $\nu$ as in proposition 5. If $b$ is the $\sigma(0)$ iteration path from $z_{0}$ to $z_{1}$, and $\xi \cdot c(b) \neq 0$, then the quantities,

$$
\frac{\left|\zeta_{k}\right|}{\omega_{W}^{k P}}, \quad \frac{\left|\zeta_{k}\right|}{s_{k}^{\mu}}
$$

also have finite limits, with $\mu$ as in proposition 5. For each of the above limits, the fractional remainder vanishes as the $k$ th power of a quantity of magnitude less than unity.

The proof of proposition 6 is presented in Appendix B.

\section{$5 \quad$ Examples}

In this final section, we examine a number of examples of kicked oscillator models with quadratic irrational parameters, covering the full range of asymptotic long-time behaviours of the aperiodic orbits. All of our numerical calculations were done using only integer arithmetic and exact algebraic operations on quadratic irrationals. The Mathematica notebooks of the electronic supplement to [24] provided a convenient starting point for the investigations. The interested reader will find details of our calculations in the electronic supplement to this article [13].

In each of our selected examples we calculate explicitly the level- $L$ displacements $d_{j}(L)$. In one of the cases we find that the $d_{j}(L)$ are independent of $L$, corresponding to uniformly bounded aperiodic orbits. In the remaining examples, we calculate the global recursion matrix $M$, reduce it to Jordan canonical form, and verify the hypotheses of the corollary to proposition 5, thus establishing the characteristic asymptotic power-law behaviour of those models. In each case, we then select a specific aperiodic point with low-period symbol sequence and verify the hypotheses of proposition 6 to establish the asymptotic self-similarity of those orbits.

In addition to the examples presented in this section, we have investigated in a similar manner the remaining models of [24]. A summary of our results is displayed in table 1. 


\subsection{Example: $\lambda=-\sqrt{2}$, corner origin}

As our first example, we consider one of the four quadratic cases with corner origin $(\lambda=$ $\pm \sqrt{2},(1 \pm \sqrt{5}) / 2$ ) for which the lifted aperiodic orbits of the residual set are bounded. The case $\lambda=-\sqrt{2}$ has a particularly simple recursive structure, and the plane decomposes into $W$-invariant quartets of connected polygonal regions ("supertiles"). The placement of the origin at a corner of the fundamental cell $\Omega$ is crucial; below we will also consider the case of a centrally placed origin, for which the aperiodic orbits are unbounded.

From [24] we learn that the scaling domain of the $\lambda=-\sqrt{2}$ model is a triangle with two polygonal return-map sub-domains, labeled by $j=0,1$. The corresponding level- $L$ to level- $(L+1)$ return paths are

$$
p(0)=(1,0,0,0,0,1,0) \quad p(1)=(1,0,0,0,0,1,0,1,0,1,0,1,0) .
$$

The level- $L$ return times are

$$
T_{0}(L)=2 \cdot 9^{L+1}+1 \equiv 3(\bmod 4) \quad T_{1}(L)=4 \cdot 9^{L+1}-2 \equiv 2(\bmod 4) .
$$

The respective $K$ and $W$-orbits of sub-domain $\mathcal{D}_{0}(0)$ are shown in figures 5 and 6 . We see that the level-0 lattice displacement vector $d_{0}(0)$ is $(0,-1)$ (recall that a factor $\tau=-\sqrt{2} / 2$ has been factored out to obtain integer lattice coordinates). A similar calculation gives $d_{1}(0)=(1,-1)$. Knowing the return paths and return times, we can now calculate $d(L)$ for arbitrary $L$ from $(21)$

$$
d(L)=(-1,-1+i)
$$

where we have used the complex representation (17). The $L$-independence of the lattice displacements corresponds to the uniform boundedness of all $W$-orbits originating in the fundamental cell $\Omega$. Consider the return-map orbits of not only $\mathcal{D}_{0}(0)$ and $\mathcal{D}_{1}(0)$, but also their translates by $(0,-\tau),(\tau,-\tau)$ and $(\tau, 0)$. The orbits are entirely contained in the fundamental cell (labeled 0$)$ ) and its 3 neighbouring cells labeled $-1,-1+i$ and $i$. Moreover, a single application of the return map $\sigma(0)$ maps $\left[\mathcal{D}_{0}(0), 0\right],\left[\mathcal{D}_{1}(0), 0\right]$ and their translates onto the scaling domains of $[\mathcal{D}(0), \zeta], \quad \zeta=0,-1,-1+i, i$, apart from discontinuity lines. Thus further iteration of the return map will never leave the region already covered by the lowest-level return orbits. Combining these orbits with a finite number of $W$-periodic octagonal domains produces what we have called a supertile, shown in figure 7 . It is easy to show that, apart from discontinuity lines, the entire plane is partitioned into invariant quartets of supertiles (in addition to the single invariant one containing $\Omega$ ).

The case $\lambda=(1-\sqrt{5}) / 2$ is similar to the present one. The cases $\lambda=\sqrt{2}$ and $\lambda=(1+\sqrt{5}) / 2$ are similar, except for the fact that the invariant sets are not connected.

\subsection{Example: $\lambda=-(1+\sqrt{5}) / 2$}

The preceding example exhibits the ultimate in stickiness: no orbit can wander very far from its initial point. Now let us consider the opposite extreme, where typical aperiodic 


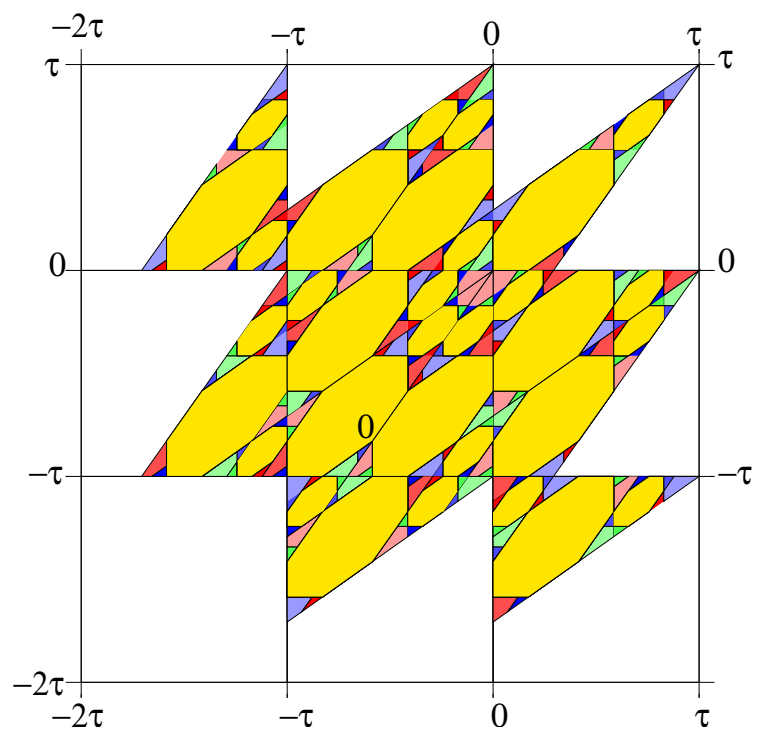

Figure 7: $W$-invariant "supertile" for $\lambda=-\sqrt{2}$.

orbits in the residual set escape to infinity with displacement asymptotically proportional to the time $(\mu=1)$.

According to [24], the scaling domain for $\lambda=-(1+\sqrt{5}) / 2$ has 3 polygonal sub-domains, which we label $j=0,1,2$. The return paths and level- $L$ return times are

$$
\begin{array}{cc}
p(0)=(1,0) \quad p(1)=(1,0,2,0) & p(2)=(1,0,2,0,2,0,2,0) \\
T_{0}(L)=\frac{2}{3}\left(4+5 \times 4^{L}\right) \equiv 0(\bmod 4), & T_{1}(L)=10 \times 4^{L} \equiv 0(\bmod 4) \\
T_{2}(L)=\frac{2}{3}\left(-8+35 \times 4^{L}\right) \equiv 0(\bmod 4) .
\end{array}
$$

Direct calculation of the level-0 orbits of $W$ gives

$$
d(0)=(-2+i,-3+i,-5+i) .
$$

The Jordan decomposition of the global recursion matrix $M=S \mathcal{M} S^{-1}$ gives

$$
S=\left(\begin{array}{ccc}
-1 & -1 & 1 \\
1 & 0 & 3 \\
1 & 2 & 7
\end{array}\right) \quad \mathcal{M}=\left(\begin{array}{lll}
0 & 0 & 0 \\
0 & 1 & 0 \\
0 & 0 & 4
\end{array}\right) \quad S^{-1}=\frac{1}{12}\left(\begin{array}{ccc}
-6 & 9 & -3 \\
-4 & -8 & 4 \\
2 & 1 & 1
\end{array}\right)
$$

hence

$$
\xi=\left(-1+\frac{i}{3}\right)(1,3,7)
$$

Explicit calculation of $\xi \cdot c(p(j \mid i))$ for all partial iteration paths verifies the hypothesis of corollary 5 , thus establishing the $\omega_{W}^{L}$ growth of all aperiodic orbits.

For the initial point of a sample aperiodic orbit in $\Omega$, we take

$$
z_{0}=\frac{1}{22}(-13+5 \sqrt{5}, 7-\sqrt{5}) \longleftrightarrow(2,2)^{\infty} .
$$




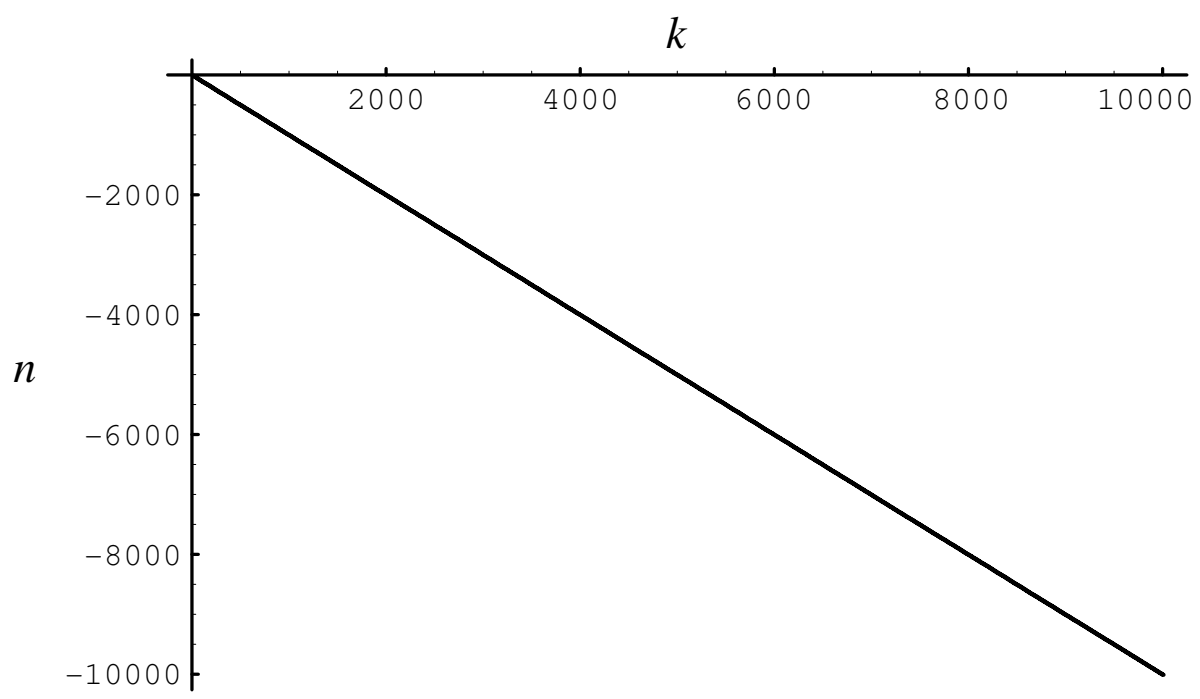

Figure 8: In the model with $\lambda=-(1+\sqrt{5}) / 2$, the vertical lattice coordinate $n=\operatorname{Re}\left(\zeta_{k}\right)$ is plotted versus iteration number $k$ for the $\sigma(0)$ orbit starting at the aperiodic point $z_{0}=\left[u_{0}, 0\right]$. Only the points $n=10 k, k=0,1, \ldots$ have been plotted. As the $W$-orbit spirals outward, the plotted sub-orbit moves downward with its horizontal coordinate never departing more than one unit from zero. The asymptotic linear increase of the distance from the origin is apparent.

Defining the symbolic substitution $\sigma: j \mapsto p(j)$, we obtain that the forward $\rho(0)$-orbit of $z_{0}$ follows the path

$$
(2) \smile a \smile \sigma(a) \smile \sigma^{2}(a) \smile \cdots \smile \sigma^{L}(a) \cdots,
$$

where $a$ is the symbol sequence $p(2 \mid 3)=(0,2,0,2,0)$. This gives for the vector $c$

$$
c(a)=(3,0,2)
$$

so that

$$
\xi \cdot c(a)=17\left(-1+\frac{i}{3}\right) \neq 0 .
$$

From proposition 6 , we have that there is a countable sequence of points $z_{L}$ on the orbit of $z_{0}$ such that $z_{L}$ scales as $4^{L}$ for $L \rightarrow \infty$. Since the temporal and global geometrical scale factors are equal, the exponent of the power-law is unity (ballistic motion for $W^{4}$ ). The orbit is displayed in figure 8 .

\subsection{Example: $\lambda=-\sqrt{3}(\mathrm{~A})$}

Of all the quadratic examples treated in [24], the only one with disjoint sequences of scaling domains is that with $\lambda=-\sqrt{3}$. The two scaling sequences, labeled $A$ and $B$, correspond to distinct limit points and temporal scale factors, as well as sets of aperiodic orbits with distinct fractal dimensions. Both are needed for a complete recursive tiling of $\Omega$. As we shall see below, the lifted aperiodic orbits of both scaling families are typically unbounded, with asymptotic power-laws having different exponents $\mu_{A}$ and $\mu_{B}$, with $\mu_{A}=\frac{1}{2}$ (diffusive) 
and $0<\mu_{B}<\frac{1}{2}$ (sub-diffusive). In the current example we deal with an $A$-orbit with a simple periodic symbol sequence.

Scaling sequence $A$ has limit point $(-\sqrt{3} / 3,-\sqrt{3} / 3)$, local geometrical scale factor $\omega_{K}=2-\sqrt{3}$, and temporal scale factor $\omega_{T}=4$. The return map partition consists of 12 polygonal sub-domains and 11 boundary line segments. For simplicity we will limit ourselves here to an aperiodic orbit which visits only 4 of the polygonal sub-domains and their higher-level partners. The sub-domains labeled 7, 10, 21, and 22 in [24] will here be designated $0,1,2,3$. Their return paths and return times are

$$
\begin{aligned}
p(0)=(3,1,0) \quad p(1) & =(3,1,1,2,0) \quad p(2)=(3,1,2,2,0) \quad p(3)=(3,2,0) \\
T_{0}(L) & =T_{3}(L)=26880 \times 4^{L}+53 \equiv 1(\bmod 4) \\
T_{1}(L) & =T_{2}(L)=53760 \times 4^{L}-53 \equiv 3(\bmod 4) .
\end{aligned}
$$

The lowest-level lattice displacement vector is

$$
d(0)=(22+310 i, 18-8 i, 18+22 i,-8+310 i)
$$

and the global recursion matrix has Jordan canonical form

$$
\mathcal{M}=\left(\begin{array}{llll}
0 & 0 & 0 & 0 \\
0 & 1 & 0 & 0 \\
0 & 0 & 1 & 0 \\
0 & 0 & 0 & 2
\end{array}\right)
$$

Explicit calculation of $\xi \cdot c(p(j \mid i))$, with

$$
\xi=328 i(1,0,0,1),
$$

for all partial iteration paths, verifies the hypothesis of the corollary, thus establishing the $\omega_{W}^{L}$ growth of all rapid-ascent orbits.

Our specific choice of aperiodic orbit starts at the point $z_{0}=\frac{1}{39}(-1422+821 \sqrt{3}, 7770-$ $4486 \sqrt{3})$ with a period-1 symbol sequence $(1,1)^{\infty}$. Defining the symbolic substitution $\sigma: j \mapsto p(j)$, we obtain that the forward $\rho(0)$-orbit of $z_{0}$ follows the path

$$
(1) \smile a \smile \sigma(a) \smile \sigma^{2}(a) \smile \cdots \smile \sigma^{L}(a) \cdots,
$$

where $a$ is the symbol sequence $(1,2,0)$. This gives for the vector $c$

$$
c(a)=(1,1, i, 0)
$$

so that

$$
\xi \cdot c(a)=328 i \neq 0 .
$$

We conclude from proposition 6 that the $W$-orbit starting at $z_{0}$ has a countable sequence of points with lattice coordinates $\zeta_{L}$ which scale as $2^{L}$, so that

$$
\zeta_{L} \sim t_{L}^{\mu} \quad \mu=\frac{\log 2}{\log 4}=\frac{1}{2}
$$

The $\sigma(0)$-orbit of $z_{0}$ is shown in figure 9 . To verify the diffusive power-law predicted by proposition 6 , we plot $\ln |\zeta|$ vs the logarithm of the iteration number in figure 10. 


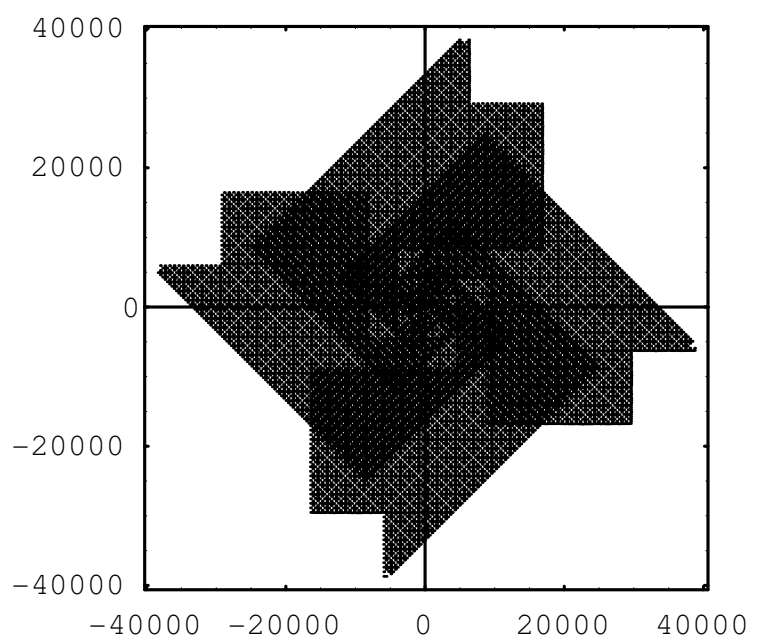

Figure 9: The first 25000 iterates of $\sigma(0)$ applied to the point $z_{0}$, for $\lambda=-\sqrt{3}$, (A).

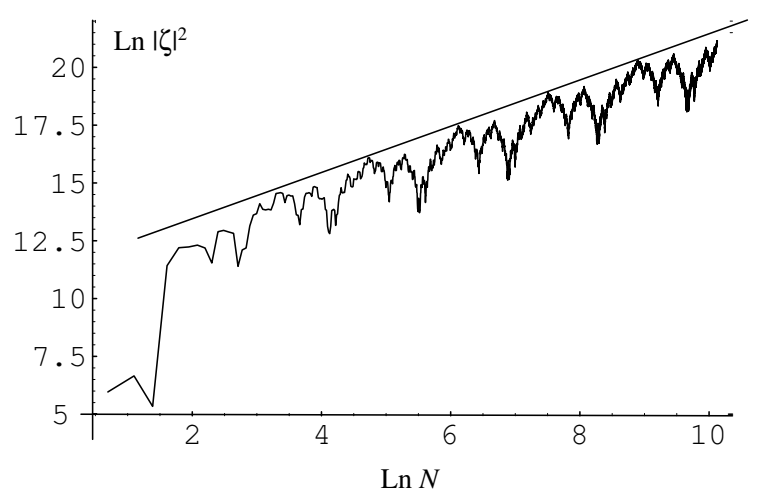

Figure 10: Log-log plot of $|\zeta|^{2}$ vs iteration number for the orbit of figure 9. The slope of the comparison line is 1 .

\subsection{Example: $\lambda=-\sqrt{3}(\mathrm{~B})$}

Scaling sequence $B$ has limit point $(41-24 \sqrt{3}, 41-24 \sqrt{3})$, local geometrical scale factor $\omega_{K}=2-\sqrt{3}$, and temporal scale factor $\omega_{T}=5$. The return map partition contains 6 polygonal sub-domains. The sub-domains labeled $0,3,5,6,8$, and 10 in [24] will here be designated $0,1,2,3,4,5$. Their return paths and return times (modulo 4) are

$$
\begin{array}{lll}
p(0)=(3) & p(1)=(3,1,4,1,2) & p(2)=(3,1,4,5,4,1,2) \\
p(3)=(3,1,4,0,4,4,1,2) & p(4)=(3,2) & p(5)=(3,2,2) \\
& & \\
T_{0}(L) \equiv 3(\bmod 4) & T_{1}(L) \equiv 0(\bmod 4) & T_{2}(L) \equiv 3(\bmod 4) \\
T_{3}(L) \equiv 3(\bmod 4) & T_{4}(L) \equiv 2(\bmod 4) & T_{5}(L) \equiv 1(\bmod 4)
\end{array}
$$

The array of level-zero displacements is

$$
d(0)=(15+25 i,-164+164 i,-15+323 i, 15+323 i, 10+10 i,-5+15 i),
$$


and the global recursion matrix has Jordan canonical form

$$
\mathcal{M}=\left(\begin{array}{cccccc}
-1 & 0 & 0 & 0 & 0 & 0 \\
0 & -1 & 0 & 0 & 0 & 0 \\
0 & 0 & 0 & 0 & 0 & 0 \\
0 & 0 & 0 & 1 & 1 & 0 \\
0 & 0 & 0 & 0 & 1 & 0 \\
0 & 0 & 0 & 0 & 0 & 2
\end{array}\right)
$$

with the complex vector $\xi$ given by

$$
\xi=164(2 i,-1+i, 4 i, 4 i, 2+2 i, 2) .
$$

Explicit calculation of $\xi \cdot c(p(j \mid i))$ for all partial iteration paths verifies the hypothesis of the corollary, thus establishing the $\omega_{W}^{L}$ growth of all aperiodic orbits.

Our specific choice of aperiodic orbit starts at the point $z_{0}$ with a symbol sequence $\left((1,2),(1,1)^{\infty}\right)$ having a period-1 tail. Defining the symbolic substitution $\sigma: j \mapsto p(j)$, we obtain that the forward $\rho(0)$-orbit of $z_{0}$ follows the path

$$
b \smile \sigma(b) \smile \sigma^{2}(b) \cdots \sigma^{L}(b) \smile \cdots,
$$

where $b$ is the symbol sequence $(4,1,2)$. This gives for the vector $c$

$$
c(b)=(0,-i, 1,0,-i, 0),
$$

so that

$$
\xi \cdot c(b)=492(1+i) \neq 0 .
$$

We conclude from proposition 5 that the $W$-orbit starting at $z_{0}$ has a countable sequence of points with lattice coordinates $\zeta_{L}$ which scale as $2^{L}$, so that

$$
\zeta_{L} \sim t_{L}^{\mu} \quad \mu=\frac{\log 2}{\log 5}<\frac{1}{2}
$$

The $\sigma(0)$-orbit of $z_{0}$ is shown in figure 11, with an accompanying log-log plot of distancesquared versus time in figure 12 .

We note that although the orbit is unbounded, with the predicted scaling $\left|\zeta_{L}\right| \sim$ $t^{\mu}, \mu=\log 2 / \log 5$, it is also recurrent, apparently returning infinitely many times to the fundamental cell. The rapid-ascent method can be exploited [13] to explore these recurrences on time scales of grandiose proportions. A histogram of the results is shown in figure 13.

\subsection{Example: $\lambda=-\sqrt{2}$, centred origin, residual set (A)}

Shifting the origin to the centre of the fundamental cell $\Omega$ can have a dramatic effect on the phase portrait of the corresponding lifted map. For $\lambda=-\sqrt{2}$, the corner-origin version has only bounded orbits (except possibly on the discontinuity set), whereas in the centre-origin 


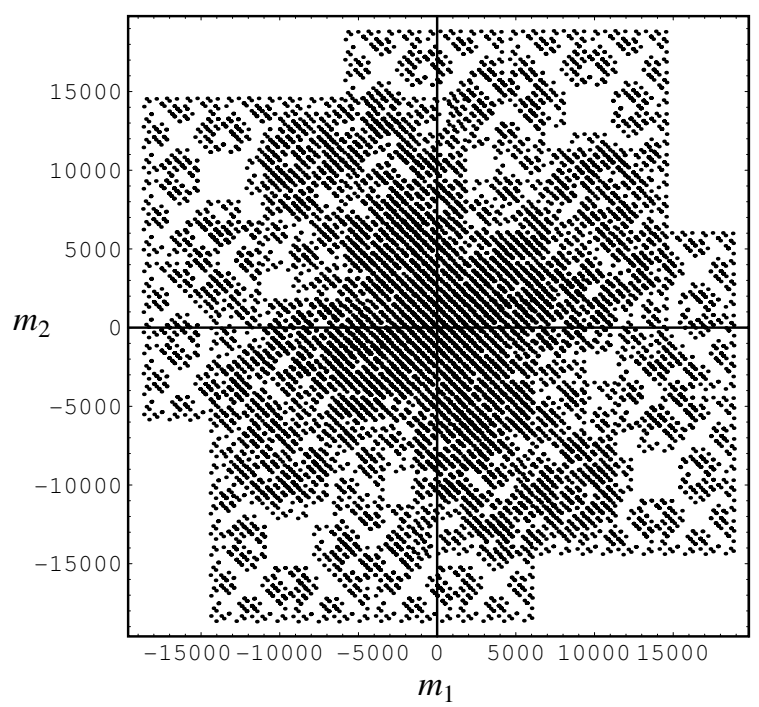

Figure 11: The first 30000 iterates of $\sigma(0)$ applied to the point $z_{0}$, for $\lambda=-\sqrt{3}$, (B).

version the aperiodic orbits in the residual set are unbounded, with sub-sequences of orbit points tending to infinity logarithmically in time. An interesting feature of this model is the remarkable distinction between orbits off and on the discontinuity set $[1,24]$. In the current example we will restrict ourselves to discontinuity-avoiding orbits, returning at the end of the section to consider the more complicated case of orbits on the discontinuity set.

Following the treatment of [24], we define the local map on the square $\Omega=(\tau,-\tau]^{2}$, where $\tau=\lambda / 2=-\sqrt{2} / 2$. The scaling sequence consists of triangles tending to the limit point $(-1-\tau, \tau)$. At each level, the scaling domain has a return-map partition with 3 polygonal sub-domains, labeled by $j=0,1,2$ (numbered $0,6,8$, respectively, in [24]). The corresponding return paths and level- $L$ return times are

$$
\begin{gathered}
p(0)=(2,0) \quad p(1)=\left(2,0,1,0,\left(1,0^{4}\right)^{2},(1,0)^{2}\right) \quad p(2)=\left(2,0,1,0,\left(1,0^{4}\right)^{5},(1,0)^{2}\right) \\
T_{0}(L)=3 \times 9^{L} \equiv 3(\bmod 4) \quad T_{1}(L)=15 \times 9^{L} \equiv 3(\bmod 4) \quad T_{2}(L)=24 \times 9^{L} \equiv 0(\bmod 4) .
\end{gathered}
$$

For the lifted map, the array of level- $L$ displacements is

$$
d(L)=(-1-(2 L+1) i,-(2 L+2) i, 2),
$$

corresponding to a complex recursion matrix

$$
M=\left(\begin{array}{ccc}
1 & 0 & -i \\
0 & 1 & -i \\
0 & 0 & 1
\end{array}\right)
$$

We note that the matrix is already in Jordan canonical form, with all diagonal elements equal to unity. The off-diagonal entries are responsible for the linear growth of $d_{j}(L)$ with increasing $L$, a property which leads to the existence of aperiodic orbits with logarithmically growing displacement from the origin. 


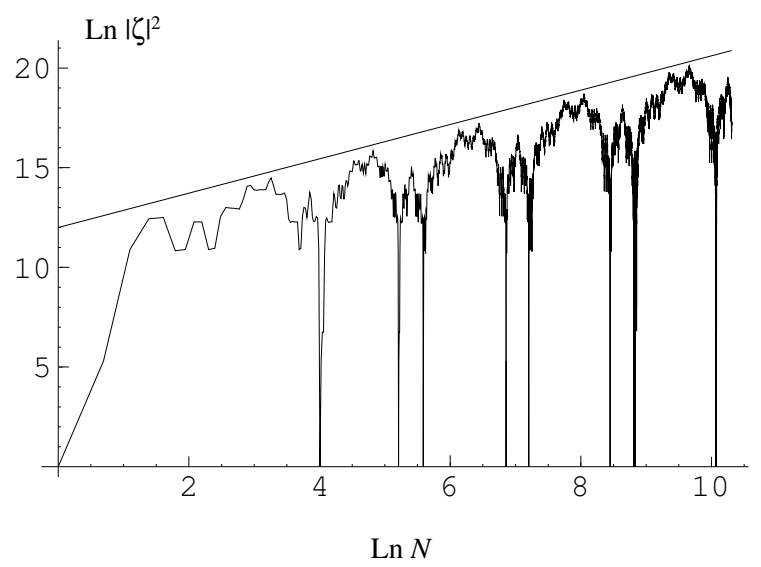

Figure 12: Log-log plot of $|\zeta|^{2}$ vs iteration number for the orbit of figure 11. The slope of the comparison line is $2 \times \frac{\log 2}{\log 5}$.

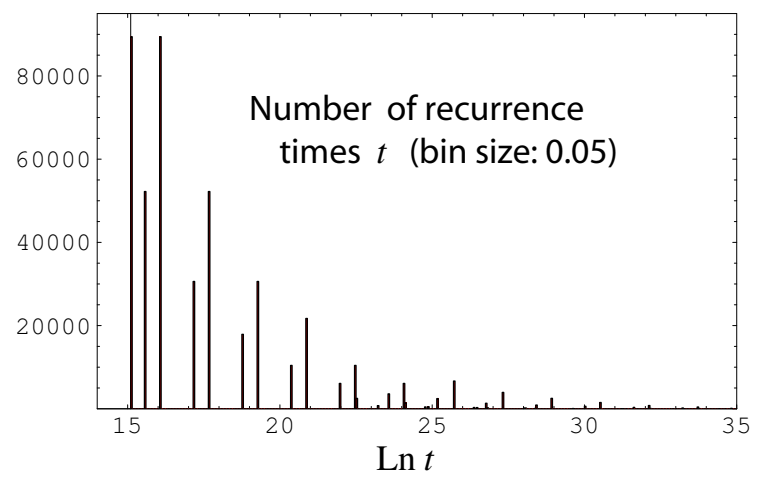

Figure 13: Histogram of recurrence times ( $W$ iterations between successive visits to the fundamental cell) for the orbit of figure 11. The abscissa is the natural logarithm of the recurrence time, and the bin size is 0.05 . The sample includes all recurrences up to time $4.73 \times 10^{22}$. For large recurrence times $t$, the frequency falls off approximately as $t^{-0.34}$.

As a specific example of an aperiodic point in the residual set, we choose the point

$$
z_{0}=\frac{1}{17}(-36+23 \sqrt{2},-4-5 \sqrt{2})
$$

whose symbolic sequence, with period-1 tail, is

$$
\left((1,3),(1,2)^{\infty}\right)
$$

The forward $\rho(0)$-orbit follows the path

$$
a \smile \sigma(a) \smile \sigma^{2}(a) \smile \cdots,
$$

where $\sigma: j \mapsto p(j)$ and $a$ is the sequence $0\left(1,0^{4}\right)^{2}(1,0)^{2}$, with corresponding coefficient vector

$$
c=(-1,1-i, 0) .
$$

Defining $\zeta_{L}$ as the lattice point attained after application of $\sigma^{L-1}(a)$ in the above sequence, we have the recursion relation

$$
\zeta_{L+1}=i \zeta_{L}-2 L-1-i
$$


Asymptotically, we have for the corresponding time (number of $W$ iterations) $t_{L} \sim 9^{L}$, and so

$$
\zeta_{L} \sim-(1+i) L \sim \log t_{L}(-1-i)
$$

In figure 14, we show the $W$-orbit of $z_{0}$, as well as a plot of $\zeta_{L}$ for a huge range of levels. Sampling the orbit on a logarithmic time scale greatly enhances our ability to see the true asymptotic behaviour.

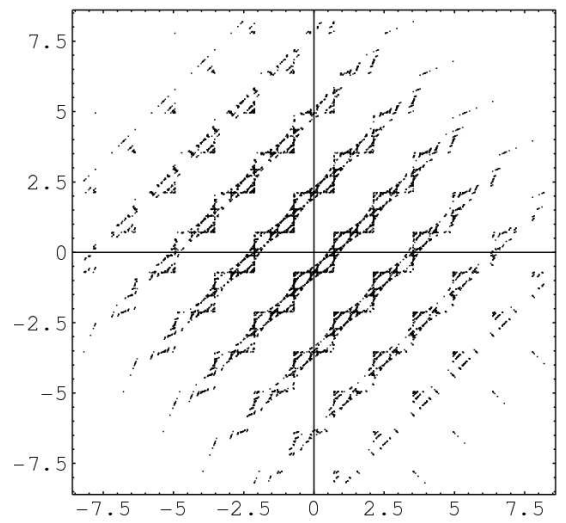

(a)

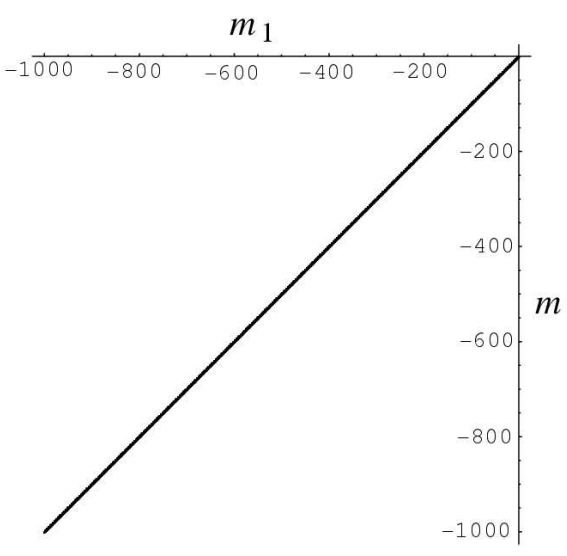

(b)

Figure 14: (a) The first 10000 iterates of $W$ applied to the point $z_{0}$, for $\lambda=-\sqrt{2}$, (A). (b) Plot of $\zeta_{L}, L=0,1, \ldots 1000$, for the same orbit.

\subsection{Example: $\lambda=-\sqrt{2}$, centred origin, discontinuity set (B)}

In this final example, we consider an aperiodic orbit on the discontinuity set. The detailed recursive structure of the latter was presented in reference [24], where it was shown that within the discontinuity set there is a Cantor set of aperiodic orbits of dimension $-\log 5 / \log (3-2 \sqrt{2})$. Thanks to a peculiar property of the global recursion matrix $M$, the lifted versions of these orbits are typically unbounded, with a super-diffusive powerlaw.

From [24], we start with the level-0 return-map partition. The nine sub-domains include the 3 polygonal ones used in the preceding example, as well as 4 open line segments and 2 points on the discontinuity set. According to the level scheme of [24], with local geometric scale factor $\omega_{K}=3-2 \sqrt{2}$, two distinct partitions apply to even and odd levels, respectively. True scaling on the discontinuity set applies separately to the even and odd level sequences, with scale factor $\omega_{K}^{2}$. For details, see the electronic supplement [13].

Direct calculation of the level-0 orbits of the global map $W$ gives us the level-0 displacements,

$$
d(0)=(-1-i, 1-5 i, 4-9 i,-7 i,-3-2 i,-2-5 i,-2 i, 5+i, 2) .
$$

From the return paths listed in [24], one obtains a temporal level-to-level scale factor $\omega_{T}=9$ and a 2-level global recursion matrix $M$ whose Jordan canonical form has a 
unique largest diagonal entry 25 , and vector $\xi$ given by

$\xi=\frac{1}{120}(0,-108-220 i,-395-318 i,-162-330 i, 54+110 i, 330-162 i, 0,-108-220 i, 0)$

For any orbit satisfying the hypotheses of proposition 6 , the asymptotic long-time behaviour is governed by a power-law with a super-diffusive exponent $\mu=\log 5 / \log 9 \approx$ 0.7325 .

Our sample orbit starts at the point

$$
z_{0}=\left(-3+\frac{9}{4} \sqrt{2}, \frac{3}{2}-\sqrt{2}\right) \in \bigcap_{L=0}^{\infty} \mathcal{D}_{7}^{(32,2)^{L}}
$$

and so has a period-2 symbol sequence $((7,32),(7,2))^{\infty}$. As in previous examples, the $\rho(0)$-orbit of $z_{0}$ is generated by a substitution rule $\sigma: j \mapsto p(j)$, where $p(j)$ is the return path for $\mathcal{D}_{j}(L)$ with respect to iterations of $\rho(L-1)$. In the present case, we have distinct substitutions $\sigma_{\mathrm{e}}$ and $\sigma_{\mathrm{o}}$ for even and odd levels. Using the Vershik symbolic dynamics, we find the following sequence of $\rho_{j}$ maps for the orbit starting at $z_{0}$ :

$$
\begin{aligned}
(7) \smile a \smile \sigma_{\mathrm{o}}(b) & \smile \sigma_{\mathrm{o}}\left(\sigma_{\mathrm{e}}(a)\right) \smile \sigma_{\mathrm{o}}\left(\sigma_{\mathrm{e}}\left(\sigma_{\mathrm{o}}(b)\right)\right) \smile \ldots \\
& \smile\left(\sigma_{\mathrm{o}} \circ \sigma_{\mathrm{e}}\right)^{L}(a) \smile\left(\sigma_{\mathrm{o}} \circ \sigma_{\mathrm{e}}\right)^{L} \circ \sigma_{\mathrm{o}}(b) \smile \ldots
\end{aligned}
$$

where

$$
a=(0) \quad b=(0,0,0,0,5,0,6,0,6,0,1,0,0,0,0,3,0,6,0,0,0,0,6,0,0,0,0,6,0,6,0) .
$$

Let $\zeta_{2 L}$ be the lattice point attained after the subsequence $\left(\sigma_{\mathrm{o}} \circ \sigma_{\mathrm{e}}\right)^{L-1} \circ \sigma_{\mathrm{o}}(b)$. Because of the repetition of the symbol sequence in alternating levels, we have a recursion relation of the form

$$
\zeta_{2 L+2}=i^{\nu} m_{2 L}+\sum_{j} c_{j} d_{j}(2 L)
$$

By explicit calculation for $L=1$ we find

$$
\nu=0 \quad c=(-10+6 i, 6 i, 0,2 i, 0,6 i, 4-4 i, 2,3 i) .
$$

Since

$$
c \cdot \xi=\frac{1}{15}(342+71 i) \neq 0
$$

we know from proposition 6 that $\zeta_{2 L}$ scales asymptotically as $5^{2 L}$. Since the time $t_{2 L}$ (number of $W$-iterations) scales as $9^{2 L}$, we have

$$
\zeta_{2 L} \sim t_{2 L}^{\mu} \quad \mu=\frac{\log 5}{\log 9} .
$$

The $\sigma(0)$-orbit of $z_{0}$ is shown in figure 15. The intermittent character of the orbit, with an alternation of rapid "flights" and long periods of localized motion, is evident in the figure and the accompanying log-log graph of distance versus iteration number. Since the orbit is located on the discontinuity set, the calculations are exceedingly sensitive to any sort of round-off error, which is quite capable of producing spurious flights. The use of exact arithmetic of algebraic numbers avoids this problem. 

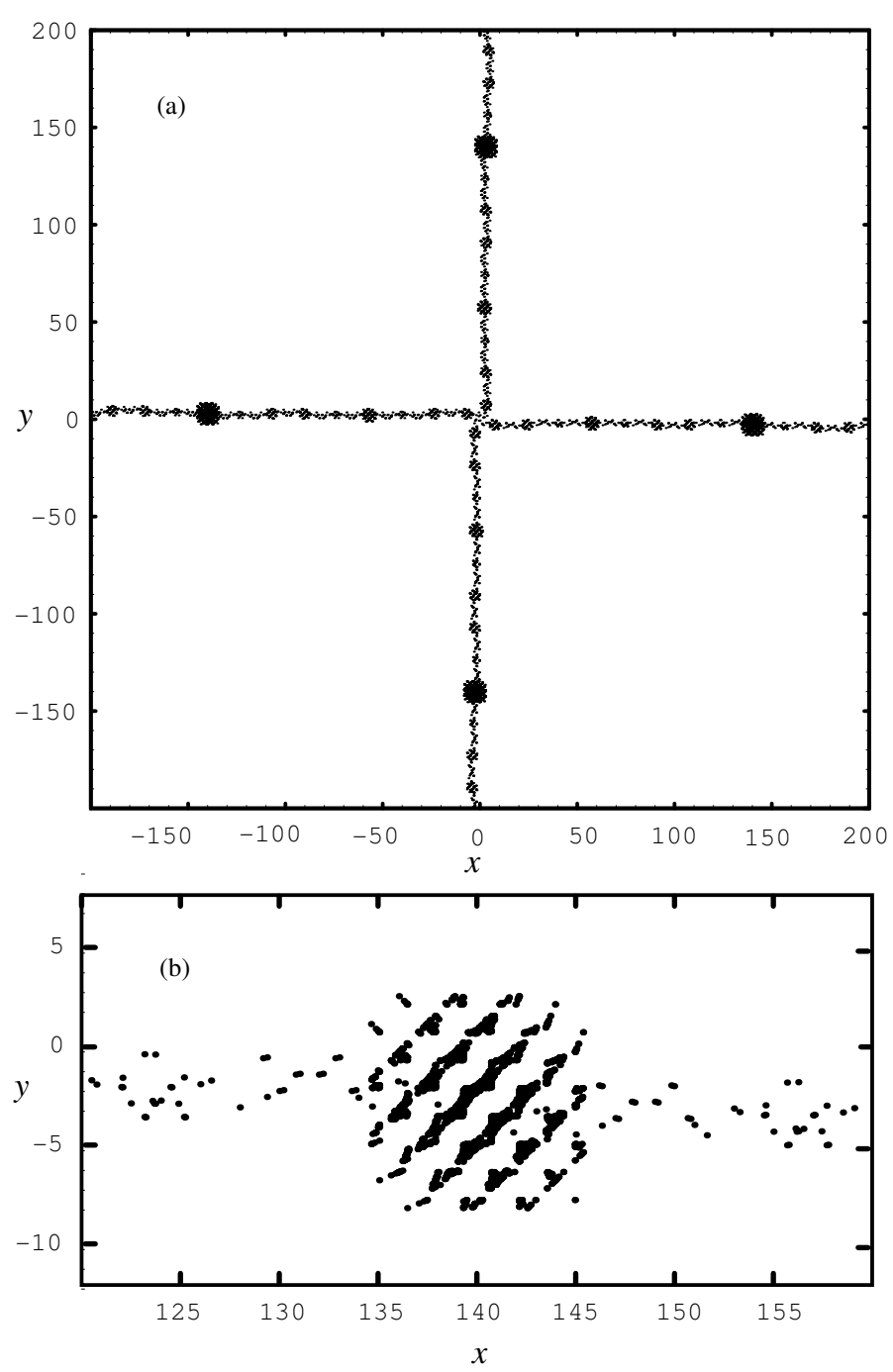

Figure 15: Top: the first 20000 iterates of $W$ applied to the point $z_{0}$, for $\lambda=-\sqrt{2}$; Bottom: detail.

\subsection{Summary of results for quadratic cases}

Table 1 summarizes the parameters and derived scale factors and dimensions of the 9 kicked-oscillator models lifted from the class of piecewise affine maps studied systematically in reference [24]. The local quantities are taken from that source, with the global scale factor $\omega_{W}$ and expansion exponent $\mu$ calculated as in the current section.

\section{Appendix A: proof of proposition 5}

To construct the rapid-ascent sub-orbit systematically, we employ the calendrical rules of symbolic updating (Vershik map). We start with the symbolic representation $\left(\left(j_{0}, t_{0}\right),\left(j_{1}, t_{1}\right), \ldots\right)$ 


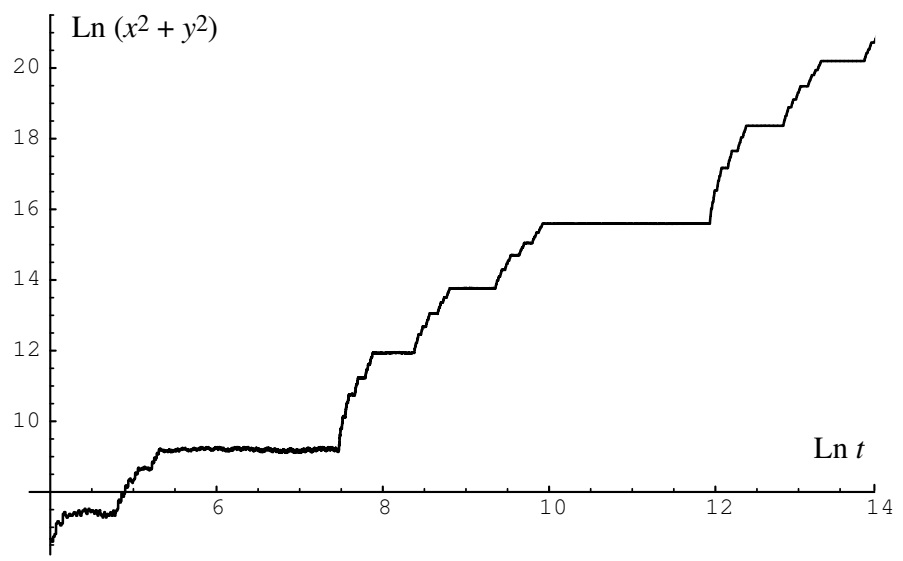

Figure 16: Log-log plot of distance-squared vs iteration number for the same orbit as in figure 15, for 1.2 million iterations.

\begin{tabular}{|c|c|c|c|c|c|}
\hline$\lambda$ & $\omega$ & $\omega_{T}$ & $\omega_{W}$ & $\mu$ & behaviour \\
\hline$\sqrt{2}$ & $3-2 \sqrt{2}$ & 9 & 1 & 0 & bounded \\
$-\sqrt{2}$ & $3-2 \sqrt{2}$ & 9 & 1 & 0 & bounded \\
$(1+\sqrt{5}) / 2$ & $(3-\sqrt{5}) / 2$ & 4 & 1 & 0 & bounded \\
$(1-\sqrt{5}) / 2$ & $(3-\sqrt{5}) / 2$ & 4 & 1 & 0 & bounded \\
$(-1+\sqrt{5}) / 2$ & $(3-\sqrt{5}) / 2$ & 4 & 4 & 1 & ballistic \\
$(-1-\sqrt{5})$ & $(3-\sqrt{5}) / 2$ & 4 & 4 & 1 & ballistic \\
$\sqrt{3}$ & $7-4 \sqrt{3}$ & 25 & 4 & .430677 & sub-diffusive \\
$-\sqrt{3}(\mathrm{~A})$ & $2-\sqrt{3}$ & 4 & 2 & .5 & diffusive \\
$-\sqrt{3}(\mathrm{~B})$ & $2-\sqrt{3}$ & 5 & 2 & .4306770 & sub-diffusive \\
$-\sqrt{2}$ (A) & $3-2 \sqrt{2}$ & 9 & 1 & 0 & logarithmic \\
$-\sqrt{2}$ (B) & $3-2 \sqrt{2}$ & 9 & 5 & .732487 & super-diffusive \\
\hline
\end{tabular}

Table 1: Summary of local and global scaling parameters for the quadratic kicked-oscillator models.

of the initial point $z$ and apply the Vershik map repeatedly. We exclude, for the time being, the possibility that the code for $z$ has a maximal tail, i.e., that $z$ is a preimage, under iteration of $W$, of a scaling limit point. After $\nu_{0}-t_{0}-1$ iterations, $t_{0}$ has reached its maximum, $\nu_{0}-1$. One additional iteration brings us to

$$
\begin{aligned}
& z_{0}=W^{\nu_{0}-t_{0}} u \longleftrightarrow \\
& \left(\left(j_{0}^{\prime}, 0\right), \ldots,\left(j_{L_{0}-1}^{\prime}, 0\right),\left(j_{L_{0}}^{\prime}, 0\right),\left(j_{L_{0}+1}, t_{L_{0}+1}+1\right),\left(j_{L_{0}+2}, t_{L_{0}+2}\right), \ldots\right)
\end{aligned}
$$

where $L_{0}$ is a non-negative integer and $j_{0}^{\prime}, \ldots, j_{L_{0}}^{\prime}$ are determined by the path conditions. Since $p(j, 0)$ is independent of $j$, we have

$$
j_{0}^{\prime}=j_{1}^{\prime}=\cdots=j_{L_{0}-1}^{\prime} .
$$

Geometrically, $\left(\nu_{0}-t_{0}\right)$-fold iteration of $W$ has brought our orbit to a point whose local coordinate $u_{0}$ lies in the level- $L_{0}$ scaling domain $\mathcal{D}\left(L_{0}\right)$. Continued $W$-iteration leads 
eventually to a symbol sequence whose $t_{i}$ are maximal up to and including $i=L_{0}+1$, so that one more iteration takes the orbit to

$$
\begin{aligned}
& z_{1}=\left[u_{1}, \zeta_{1}\right]=\sigma\left(L_{0}\right)^{\nu_{L_{0}}-t_{L_{0}}-1} z_{0} \longleftrightarrow \\
& \left(\left(j_{0}^{\prime}, 0\right), \ldots,\left(j_{0}^{\prime}, 0\right),\left(p\left(j_{L_{1}+1}, t_{L_{1}+1}+1\right), 0\right),\left(j_{L_{1}+1}, t_{L_{1}+1}+1\right),\left(j_{L_{1}+2}, t_{L_{1}+2}\right), \ldots\right),
\end{aligned}
$$

where $L_{1}>L_{0}$ and $u_{1} \in \mathcal{D}\left(L_{1}\right)$. We note that the partial return path for the iterated application of $\sigma\left(L_{0}\right)$ is just $p\left(j_{L_{0}} \mid t_{L_{0}+1}\right)$, defined in $(22)$.

Repetition of the above procedure generates an infinite sequence of points $\left(z_{0}, z_{1}, \ldots\right)$, with $z_{k}=\left[u_{k}, \zeta_{k}\right], u_{k} \in \mathcal{D}\left(L_{k}\right), L_{k+1}>L_{k}$. This is the rapid ascent orbit of the proposition.

If it turns out that $z$ is a preimage of a point with symbolic representation $(j, 0)^{\infty}$, we take instead the rapid ascent sub-orbit of the scaling limit point itself, since that point's code has a non-maximal tail and we can proceed with the Vershik updating as described above.

We now proceed to verify the properties $(i-v)$.

Temporal scaling. From lemma 3 we have that $T_{j}(L) \omega_{T}^{-L}$ is bounded above and below, uniformly in $L$ and $j$. Moreover, we have the following recursion relation for times on a rapid-ascent orbit:

$$
s_{k+1}=s_{k}+\sum_{t=t_{L_{k}+1}+1}^{\nu_{L_{k}+1}-1} T_{p\left(j_{L_{k}+1}, t\right)}\left(L_{k}\right)
$$

where the sum, divided by $\omega_{T}^{L_{k-1}}$, is uniformly bounded above and below by positive constants $\gamma_{ \pm}$. Thus

$$
\beta_{-} \stackrel{\text { def }}{=} \gamma_{-}<s_{k} \omega_{T}^{-L_{k-1}}<\gamma_{+} \omega_{T}^{-L_{k-1}}\left(\omega_{T}^{L_{0}}+\omega_{T}^{L_{1}}+\cdots+\omega_{T}^{L_{k-1}}\right)<\gamma_{+} \sum_{k=0}^{\infty} \omega_{T}^{-k} \stackrel{\text { def }}{=} \beta_{+},
$$

with $\beta_{ \pm}$independent of $k$.

Local power law. The local scaling limit $u_{\infty}$ belongs to the closure of all $\mathcal{D}\left(L_{k}\right)$, and hence

$$
|| u_{k}-u_{\infty}|| \leq\left|\mathcal{D}\left(L_{k}\right)\right|=\omega^{L_{k}}|\mathcal{D}(0)|<|\mathcal{D}(0)| \omega^{L_{k-1}} .
$$

Combining this with the inequalities

$$
\frac{s_{k}}{\beta_{+}}<\omega_{T}^{L_{k-1}}<\frac{s_{k}}{\beta_{-}}
$$

gives us the local asymptotic power law.

Global power law. The successive lattice points satisfy the recursion relation

$$
\zeta_{k+1}=i^{m_{k}} \zeta_{k}+c\left(p\left(j_{L_{k}} \mid t_{L_{k}}\right)\right) \cdot d(L),
$$


where

$$
m_{k}=T\left(p\left(j_{L_{k}} \mid t_{L_{k}}\right)\right)
$$

The partial paths $p(j \mid t)$ were defined in equation (22), and, from (24),

$$
\left|c\left(p\left(j_{L} \mid t_{L}\right)\right) \cdot d(L)\right|=\left|c\left(p\left(j_{L} \mid t_{L}\right)\right) \cdot \xi\right| \omega_{W}^{L}\left(1+O\left(\delta^{L}\right)\right),
$$

for some $\delta<1$. Because there are only finitely many distinct partial paths, there exist positive $\alpha_{ \pm}$such that for all $L$ with non-vanishing $c\left(p\left(j_{L} \mid t_{L}\right)\right) \cdot \xi$,

$$
\alpha_{-} \leq\left|\left(c\left(p\left(j_{L} \mid t_{L}\right)\right) \cdot \xi\right)\right| \leq \alpha_{+} .
$$

Thus

$$
\frac{\left|\zeta_{k}\right|}{\omega_{W}^{L_{k-1}}} \leq \alpha_{+} \sum_{j=0}^{k-1} \omega_{W}^{L_{j}-L_{k-1}}\left(1+O\left(\delta^{L_{j}}\right)\right) .
$$

On the right hand side the summation can be extended to infinity to obtain a $k$-independent upper bound for $\left|\zeta_{k}\right| / \omega_{W}^{L_{k-1}}$, and so we get

$$
\varlimsup_{L \rightarrow \infty} \frac{\left|\zeta_{L}\right|}{\omega_{W}^{L}}<\infty
$$

Finally we must show that the $\varlimsup$ im is non-zero. To see this, choose an arbitrary positive $\kappa$ less than $\frac{\alpha_{-}}{2 \omega_{W}}$. We claim that

$$
\varlimsup \frac{\left|\zeta_{k}\right|}{\omega_{W}^{L_{k-1}}}>\frac{\alpha_{-}}{2 \omega_{W}}-\kappa
$$

Suppose the contrary. In that case, there are infinitely many $k$ for which $\left|\zeta_{L_{k}} / \omega_{W}^{L_{k}}\right| \leq$ $\alpha_{-} /\left(2 \omega_{W}\right)-\kappa$. But then the next level lattice displacement, $\zeta_{k+1}$, would have to satisfy

$$
\frac{\left|\zeta_{k+1}\right|}{\omega_{W}^{L_{k}}} \geq \frac{\alpha_{-}}{\omega_{W}}\left(1+O\left(\delta^{L_{k}}\right)\right)-\left(\frac{\alpha_{-}}{2 \omega_{W}}-\kappa\right)=\frac{\alpha_{-}}{2 \omega_{W}}+\kappa+\frac{\alpha_{-}}{\omega_{W}} O\left(\delta^{L_{k}}\right) .
$$

For sufficiently large $k$, these quantities exceed the hypothetical $\overline{\lim }$, and, since there are infinitely many of them, we have a contradiction. We conclude that $\overline{\lim } \frac{\left|\zeta_{k}\right|}{\omega_{W}^{L}}$ is strictly positive.

Inserting the temporal scaling inequality, we obtain the global asymptotic power law.

\section{Appendix B: proof of proposition 6}

The eventual self-similarity of the forward $\sigma(0)$ orbit of any aperiodic point $z \in \mathcal{D}(0)$ is essentially a consequence of the structure of the iteration path, i.e., the sequence of $j \in I_{J}$ 
corresponding to the domains $\mathcal{D}_{j}(0)$ visited by the orbit. From the discussion of the Vershik updating in Appendix A, it is clear that the full path $g$ can be written, assuming that the symbolic representation of $z$ does not have a maximal tail, as a concatenation (denoted by the symbol $\smile$ ) of segments of the form

$$
g_{1}=p\left(j_{1} \mid t_{1}\right), \quad g_{L}= \begin{cases}0 & \text { if } t_{L}=\nu_{j_{L}}-1, \\ \mathcal{S}^{L-1} p\left(j_{L} \mid t_{L}+1\right) & \text { otherwise }\end{cases}
$$

where $\mathcal{S}$ is the substitution

$$
\mathcal{S}\left(i_{1}, i_{2}, \ldots, i_{N}\right)=p\left(i_{1}\right) \smile p\left(i_{2}\right) \smile \cdots \smile p\left(i_{N}\right),
$$

with $p(j)$ and $p(j \mid t)$ the path functions defined by (12) and (22). Thus, if the symbolic representation of the starting point $z$ is $P$-periodic from level $L^{\prime}$ onward, then we have

$$
g=a \smile b \smile \mathcal{S}^{P} b \smile \mathcal{S}^{2 P} b \smile \cdots,
$$

where

$$
a=g_{1} \smile g_{2} \smile \cdots \smile g_{L^{\prime}-1}, \quad b=g_{L^{\prime}} \smile \cdots \smile g_{L^{\prime}+P-1} .
$$

But this is only the simplest decomposition of the path consistent with eventual selfsimilarity. More generally, we can choose an arbitrary initial segment $h$ of $g_{L^{\prime}}$, including $h=\emptyset$. Then (29) remains valid with

$$
a=g_{1} \smile g_{2} \smile \cdots \smile g_{L^{\prime}-1} \smile h, \quad b=\left(g_{L^{\prime}} \backslash h\right) \smile \cdots \smile g_{L^{\prime}+P-1} \smile \mathcal{S}^{P} h .
$$

In the above we assumed that $z$ does not have a maximal tail. Otherwise we know that the forward orbit contains a point $z^{\prime}$ with symbolic representation $(j, 0)^{\infty}$, with $j=p(j, 0)$. Unless this point is its own successor (forbidden by our assumption that $z$ is an aperiodic point), the code does not have a maximal tail and we can apply the decomposition (29) to $z^{\prime}$ instead of $z$. The non-maximality assumption guarantees that the path segment $b$ in (30) is non-empty, since either $h \neq \emptyset$, hence $\mathcal{S}^{P} h \neq \emptyset$, or at least one of the $g_{L}, L^{\prime}<L<L^{\prime}+P-1$ is non-empty.

We now are in a position to construct the rapid-ascent sub-orbits of the proposition. Given $z$, we define, for $k=0,1, \ldots$,

$$
z_{k}=W^{s_{k}} z_{0}, \quad s_{0}=s(a), \quad s_{k}=s\left(a \smile b \smile \mathcal{S} b \smile \cdots \smile \mathcal{S}^{(k-1) P} b\right), \quad k>0,
$$

where, for any path segment $f$, the timekeeping function $s(f)$ is defined as

$$
s(f)=\sum_{j=0}^{J-1} n_{j}(f) T_{j}(0),
$$

with $n_{j}(f)$ denoting the number of elements of $f$ equal to $j$. Thus, for any non-negative integer $L$,

$$
s\left(\mathcal{S}^{L} f\right)=\sum_{j=0}^{J-1} n_{j}(f) T_{j}(L) .
$$

We now verify the claimed properties of the sequence $\left(z_{0}, z_{1}, \ldots\right)$. 
Temporal scaling. From the bounds on $T_{j}(L)$ in lemma 3 we get that there exist positive constants $B(b)$ and $C(b)$ such that, for any non-negative integer $n$,

$$
\left|s\left(\mathcal{S}^{n} b\right)-B(b) \omega_{T}^{n P}\right|<C(b) \omega^{\prime n P} .
$$

Summing up, we get that there exist positive constants $B$ and $C$, as well as $\omega^{\prime}<\omega_{T}$ such that for all $k$,

$$
\left|s_{k}-B \omega_{T}^{k P}\right|<C \omega^{\prime k P} .
$$

The finiteness of $\lim s_{k} / \omega_{t}^{k P}$ follows immediately.

Local scaling. We note that $u_{0} \in \mathcal{D}(0)$. After completing the part of the orbit with path $a$, the orbit point is still in $\mathcal{D}(0)$. We cannot say more than that: even though the orbit achieved level $L^{\prime}-1$ along the way, the arbitrariness of $h$ means that the level may fall to zero by the end of the segment. By the same token, the ultimate level of $u_{k}$, governed by the final segment $\mathcal{S}^{k P} h$, will be at least $k P$. The scaling relation

$$
|\mathcal{D}(k P)|=|\mathcal{D}(0)| \omega^{k P},
$$

together with the fact that both $u_{k}$ and $u_{\infty}$ are in the closure of $\mathcal{D}(k P)$, gives us the finiteness of $\lim _{k \rightarrow \infty}\left\|u_{k}-u_{\infty}\right\| / \omega^{k P}$. Inserting (31) gives us the power law for local convergence.

Global scaling. From the path decomposition (29), we have the lattice recursion relation for the sub-orbit $\left(z_{0}, z_{1}, \ldots\right), z_{k}=\left[u_{k}, \zeta_{k}\right]$,

$$
\zeta_{k+1}=i^{s_{k+1}-s_{k}} \zeta_{k}+c(b) \cdot d(k P) .
$$

Applying (24), the recursion formula becomes

$$
\zeta_{k+1}=i^{s_{1}-s_{0}} \zeta_{k}+c(b) \cdot \xi \omega_{W}^{k P}+O\left(\omega^{\prime k P}\right)
$$

with $\omega^{\prime}<\omega_{W}$. Thus

$$
\frac{\zeta_{k}}{\omega_{W}^{k P}}=c(b) \omega^{-P}\left(1+i^{s_{1}-s_{0}} \omega_{W}^{-P}+i^{2\left(s_{1}-s_{0}\right)} \omega_{W}^{-2 P}+\cdots+i^{(k-1)\left(s_{1}-s_{0}\right)} \omega_{W}^{-(k-1) P}\right)+r(k),
$$

where $r(k)$ vanishes as $O\left(\omega^{\prime} / \omega_{W}\right)$ if $\omega^{\prime}>1$, and $O(1)$ otherwise. Given the non-vanishing of $c(b)$, the existence of a finite, non-zero limit follows. Inserting (31) gives us the power law for global convergence.

\section{Acknowledgements}

One of us (J.H.L) would like to thank G. Zaslavsky for helpful discussions. This research was supported by EPSRC grant No GR/S62802/01. 


\section{References}

[1] R. Adler, B. Kitchens and C. Tresser, Dynamics of nonergodic piecewise affine maps of the torus, Ergod. Th. and Dynam. Sys. 21 (2001) 959-999.

[2] V. Afraimovich, A. Maass, and J. Urías, Symbolic dynamics for sticky sets in Hamiltonian systems, Nonlinearity 132000 617-637.

[3] P. Ashwin, Elliptic behaviour in the sawtooth standard map, Phys. Lett. A 232 (1997) 409-416.

[4] P. Ashwin, W. Chambers, and G. Petrov, Lossless digital filters overflow oscillations: approximations of invariant fractals, Inter. J. Bifur. Chaos 7 (1997) 2603-2610.

[5] H. Bruin, A. Lambert, G. Poggiaspalla, S. Vaienti Numerical Investigations of a Discontinuous Rotation of the Torus, Chaos 13, (2003), no.2, 558-571.

[6] J. Buzzi, Piecewise isometries have zero topological entropy, Ergod. Th. and Dynam. Sys. 21 (2001) 1371-1377.

[7] B. V. Chirikov, A universal instability of many-dimensional oscillator systems, Phys. Reports 52 (1979) 263-379.

[8] B.V. Chirikov and F.Vivaldi, An algorithmic view of pseudochaos Physica D $\mathbf{1 2 9}$ (1999) 223-235.

[9] L. O. Chua and T. Lin, Chaos in digital filters, IEEE Trans. Circuits and Systems CAS-35 (1988) 648-658.

[10] L. O. Chua and T. Lin, Fractal pattern of second order non-linear digital filters: a new symbolic analysis, Int. J. Cir. Theor. Appl. 18 (1990) 541-550.

[11] I. Dana, Global superdiffusion of weak chaos, Phys. Rev. E 69 (2004) 016212.

[12] A. C. Davies, Nonlinear oscillations and chaos from digital filters overflow, Phil. Trans. R. Soc. Lond. A 353 (1995) 85-99.

[13] Electronic supplement to the present article: http://home.nyu.edu/ jhl2/Supplement.pdf.

[14] K. J. Falconer, Fractal geometry, Wiley, New York (1990).

[15] S. Galatolo, Complexity, initial condition sensitivity, dimension and weak chaos in dynamical systems, Nonlinearity 16 (2003) 1219-1238.

[16] A. Goetz, Dynamics of piecewise isometries, PhD Thesis, University of Chicago (1996).

[17] A. Goetz, Dynamics of a piecewise rotation, Continuous and Discrete Dyn. Sys. 4 (1998) 593-608.

[18] A. Goetz, Dynamics of piecewise isometries, Illinois Journal of Mathematics 44 (2000) $465-478$. 
[19] A. Goetz, Stability of cells in non-hyperbolic piecewise affine maps and piecewise rotations, Nonlinearity 14 (2001) 205-219.

[20] A. Goetz and G. Poggiaspalla, Rotation by $\pi / 7$, Nonlinearity 17 (2004) 1787-1802.

[21] B. Kahng, Dynamics of symplectic affine maps on tori, PhD Thesis, University of Illinois at Urbana-Champain (2000).

[22] B. Kahng, Dynamics of symplectic piecewise affine elliptic rotation maps on tori, Ergodic Theory and Dynamical Systems 22 (2002) 483-505.

[23] C. F. F. Karney, Long time correlations in the stochastic regime, Physica D 8 (1983) $360-380$.

[24] K. L. Kouptsov, J.H. Lowenstein and F. Vivaldi, Quadratic rational rotations of the torus and dual lattice maps, Nonlinearity 15 (2002) 1795-1482.

[25] J. H. Lowenstein, S. Hatjispyros and F. Vivaldi, Quasi-periodicity, global stability and scaling in a model of Hamiltonian round-off, Chaos 7 (1997) 49-66.

[26] J. H. Lowenstein, Pseudochaos for piecewise rational rotations, preprint, New York University (2003) http://home.nyu.edu/ jhl2/pseudochaos.pdf .

[27] J. H. Lowenstein, K. L. Kouptsov, and F. Vivaldi, Recursive tiling and geometry of piecewise rotations by $\pi / 7$ Nonlinearity 172004 1-25.

[28] J. H. Lowenstein and F. Vivaldi, Embedding dynamics for round-off errors near a periodic orbit, Chaos (2000) 747-755.

[29] O. Lyubomudrov, M. Edelman, and G. M. Zaslavsky, Pseudochaotic systems and their fractional kinetics, Int. J. Mod. Phys B 17 (2003) 4149-4167.

[30] J. Meiss, Class renormalization: islands around islands, Phys. Rev. A 34 (1986) $2375-2383$.

[31] J. Meiss, Transient measures in the standard map, Physica D 74 (1994) 254-267.

[32] J. Meiss and E. Ott, Markov tree model of transport in area preserving maps, Physica D 20 (1986) 387-402.

[33] G. Poggiaspalla, Self-similarity in piecewise isometric systems, preprint, (2003) http://www . maths.qmul.ac.uk/ gpp/selfsimilarity.pdf.

[34] M. Queffélec, Substitution Dynamical Systems - Spectral Analysis, Lecture Notes in Mathematics, Vol. 1294 (1987).

[35] C. W. Wu and L. O. Chua, Properties of admissible sequences in a second-order digital filter with overflow non-linearity, Int. J. Cir. Theor. Appl. 21 (1993) 299-307.

[36] G. M. Zaslavskii, R. Z. Sagdeev, D. A. Usikov, and A. A. Chernikov, Weak chaos and quasiregular patterns, Cambridge University Press, Cambridge (1991).

[37] G. M. Zaslavsky, Physics of Chaos in Hamiltonian Systems Imperial College Press, London (1998). 
[38] G. M. Zaslavsky, M. Edelman, and B. A. Nyazov, Self-similarity, renormalization, and phase space nonuniformity of Hamiltonian chaotic dynamics, Chaos 7 (1997) $159-181$.

[39] G. M. Zaslavsky and B. A. Nyazov, Fractional kinetics and accelerator modes, Phys. Rep. 283 (1997) 73-93.

[40] G. M. Zaslavsky and M. Edelman, Weak mixing and anomalous kinetics along filamented surfaces, Chaos 11 (2001) 295-305.

[41] G. M. Zaslavsky and M. Edelman, Pseudochaos, Perspectives and Problems in Nonlinear Science: a Celebratory Volume in Honor of Lawrence Sirovich, eds. E. Kaplan, J. Marsden, and K. R. Sreenivasan, Springer, New York (2003) 421-423. 